\title{
Analytical solutions and moment analysis of general rate model for linear liquid chromatography
}

\author{
Shamsul Qamara,b,*, Javeria Nawaz Abbasib, Shumaila Javeed ${ }^{\mathrm{a}, \mathrm{b}}$, Andreas \\ Seidel-Morgenstern ${ }^{\mathrm{a}}$ \\ ${ }^{a}$ Max Planck Institute for Dynamics of Complex Technical Systems Magdeburg, Germany \\ ${ }^{b}$ Department of Mathematics, COMSATS Institute of Information Technology, \\ Park Road Chak Shahzad Islamabad, Pakistan
}

\begin{abstract}
The general rate model (GRM) is considered to be a comprehensive and reliable mathematical model for describing the separation and mass transfer processes of solutes in chromatographic columns. However, the numerical solution of model equations is complicated and time consuming. This paper presents analytical solutions of the GRM for linear adsorption isotherms and different sets of boundary conditions at the column inlet and outlet. The analytical solutions are obtained by means of Laplace transformation. Numerical Laplace inversion is used to transform back the solution in the time domain because analytical inversion cannot be obtained. The first four temporal moments are derived analytically using the Laplace domain solutions. The moments of GRM are utilized to analyze the retention times, band broadenings, front asymmetries and kurtosis of the elution profiles. Relationships are derived among the kinetic parameters to match the first four moments of GRM and the simpler lumped kinetic model (LKM). For validation, the analytical solutions are compared with numerical solutions of a second order finite volume
\end{abstract}

\footnotetext{
${ }^{*}$ Corresponding author. Tel: +49-391-6110454; fax: +49-391-6110500

Email addresses: shamsul.qamar@comsats.edu.pk (Shamsul Qamar)
} 
scheme. The good agreements in the results verify the correctness of analytical solutions and the accuracy of the numerical scheme.

Key words: Dynamics of chromatography, general rate model, analytical solutions, moments analysis, finite volume schemes.

\section{Introduction}

Column liquid chromatography is one of the most versatile separation techniques. It is widely used for analysis and purification in several industries aiming to produce pharmaceuticals, food, and fine chemicals. The concept is successfully applied to perform numerous difficult separation processes, for instance the separation of enantiomers and the isolation of specific proteins from fermentation broths. In the column liquid chromatography, a mobile phase percolates through a bed of fixed porous particles, carrying the mixture components which interact differently with the stationary phase. Components interacting strongly with the particles will be transported (elute) slowly along the column as compared to the components with weaker interactions. Therefore, each component will form a concentration band profile moving with a specific velocity in the column. These velocity differences make possible, for long enough columns, to collect pure fractions of components at the outlet of the column.

Mathematical modeling of chromatographic processes is useful for understanding and analyzing dynamic composition fronts in chromatographic columns without extensive experiments. Different mathematical models with different degrees of complexity describing the mass transfer and partition processes are available in the literature. The most important 
of these models are the general rate model (GRM), the lumped kinetic model (LKM), the equilibrium-dispersive model (EDM), and the ideal model of chromatography, see e.g. Guiochon (2003); Guiochon and Lin (2003); Guiochon et al. (2006); Ruthven (1984); Carta (1988).

The EDM assumes that the mass transfer is of infinite rate. The LKM incorporates with the rate of variation of the local concentration of solute in the stationary phase and local deviation from equilibrium concentrations. The analytical solutions and moment analysis of these models are already presented in detail in our previous publications Javeed et al. (2013); Qamar et al. (2013). In this paper, the analysis of GRM is our main concern.

The GRM is considered to be a very comprehensive model and has the potential to achieve an accurate description of chromatographic profiles. It incorporates several important factors of the mass transfer process in the column, such as the axial dispersion, external mass transfer resistance, pore diffusion and surface diffusion.

In this work, the analytical solutions of GRM are obtained for different sets of boundary conditions considering a single component fluid. The model equations are solved by using the Laplace transformation. Moment analysis has been comprehensively discussed in the literature, see for example Kubin (1964, 1965); Kucera (1965); Schneider and Smith (1968); Suzuki (1973); Wolff et al. (1979, 1980); Ruthven (1984); Lenhoff (1987); Antos (2003); Guiochon et al. (2006), Miyabe et al. (2000, 2003, 2007, 2009) and Javeed et al. (2013). In these partly classical papers analytical expressions have been generated for specific chromatographic models and boundary conditions. The analysis typically covered just the most important first and second moments, i.e. retention times and band broadening. In a 
few studies also the third moment, which describes peak and front asymmetries, was derived and evaluated. In the present paper we address several aspects that have not been treated in this detail up to now. We will derive and compare also the fourth moment, i.e. the kurtosis or flatness. Using low-noise detectors and complete capture of the responses this moment appears to be still experimentally accessible. Since the influence of the boundary conditions is often not discussed in sufficient depth, we will further compare the moment expressions for Danckwerts and Dirichlet conditions considering both rectangular pulses and steps as inlet profiles. In order to compare quantitatively the first four moments of GRM and LKM are derived. With this analysis it is intended to elucidate the connections between the specific kinetic parameters, including for the first time the results for the fourth moments. Finally, going beyond previous studies, we will provide a comparisons of the analytically derived moments with moments calculated independently by integrating numerically calculated effluent profiles. For this advanced high resolution methods are applied Javeed et al. (2011), which are capable to treat also the more general case of nonlinear equilibria.

The structure of the article is as follows: The GRM is described in Section 2. Section 3 presents the derivation of analytical solutions and moments of the GRM. Numerical test problems are presented in Section 4. Concluding remarks are given in Section 5.

\section{The General Rate Model (GRM)}

The GRM considers, besides functions for the distribution equilibria, several contributions of mass transfer processes occurring in chromatography which cause band broadening. 
More specifically, axial dispersion, mass transfer between mobile and stationary phases and intraparticle pore diffusion are included in the mass balance equations. Limiting finite rates of adsorption-desorption are sometimes also included but not considered below. Thus, the GRM contains two mass balances for each solute, one for the column and one for the particles of stationary phase.

The mass balance for a single solute component percolating through a column filled with spherical particles of radius $R_{p}$ is given as

$$
\frac{\partial c}{\partial t}+u \frac{\partial c}{\partial z}=D_{L} \frac{\partial^{2} c}{\partial z^{2}}-\frac{3}{R_{p}} F k_{e x t}\left(c-c_{p}\left(r=R_{p}\right)\right)
$$

In the above equation, $c$ and $c_{p}$ are the concentrations of a solute in the bulk of the fluid and in particle pores, respectively. The phase ratio $F$ is defined as $F=(1-\epsilon) / \epsilon$, where $\epsilon$ is the external porosity. Moreover, $u$ is the interstitial velocity, $D_{L}$ represents the axial dispersion coefficient, $k_{e x t}$ is the external mass transfer coefficient, and $t$ and $z$ denote time and axial coordinate of the column. In addition, $r$ is the radial coordinate of spherical particles of radius $R_{p}$.

The mass balance equation for the solute in the stationary phase can be expressed assuming two mechanisms of intraparticle transport:

$$
\epsilon_{p} \frac{\partial c_{p}}{\partial t}+\left(1-\epsilon_{p}\right) \frac{\partial q^{*}}{\partial t}=\frac{1}{r^{2}} \frac{\partial}{\partial r}\left(r^{2}\left[\epsilon_{p} D_{p} \frac{\partial c_{p}}{\partial r}+\left(1-\epsilon_{p}\right) D_{s} \frac{\partial q^{*}}{\partial r}\right]\right)
$$

where $q^{*}$ is the local concentration of solute in stationary phase, $\epsilon_{p}$ is the internal porosity, $D_{p}$ is the pore diffusivity, and $D_{s}$ is the surface diffusivity.

Eqs. (1) and (2) are connected at $r=R_{p}$ via the following expression which quantifies the 
temporal change of the average loading of the particles:

$$
\left[\epsilon_{p} D_{p} \frac{\partial c_{p}}{\partial r}+\left(1-\epsilon_{p}\right) D_{s} \frac{\partial q}{\partial r}\right]_{r=R_{p}}=k_{e x t}\left(c-\left.c_{p}\right|_{r=R_{p}}\right)
$$

The initial condition of the Eq. (1) for an initially regenerated column is given as

$$
c(0, z)=0, \quad(0<z<L)
$$

and initial conditions of Eq. (2) considering empty particles are given as

$$
q^{*}(0, z, r)=0, \quad c_{p}(0, z, r)=0
$$

Because rapid adsorption or desorption rates are assumed, the concentrations of solute in the pores and that in the stationary phase are in the state of equilibrium.

Only linear adsorption isotherms are considered in this work:

$$
q^{*}=a c_{p}
$$

By using Eq. (6), the right hand side term in the square brackets of Eq. (2) can be simplified as

$$
\epsilon_{p} D_{p} \frac{\partial c_{p}}{\partial r}+\left(1-\epsilon_{p}\right) D_{s} \frac{\partial q^{*}}{\partial r}=D_{e f f} \frac{\partial c_{p}}{\partial r}
$$

where

$$
D_{\text {eff }}=\epsilon_{p} D_{p}+\left(1-\epsilon_{p}\right) D_{s} a
$$

Thus, in linear from, Eq. (2) can be rewritten as

$$
a^{*} \frac{\partial c_{p}}{\partial t}=\frac{D_{e f f}}{r^{2}} \frac{\partial}{\partial r}\left(r^{2} \frac{\partial c_{p}}{\partial r}\right)
$$


with

$$
a^{*}=\epsilon_{p}+\left(1-\epsilon_{p}\right) a .
$$

Similarly, Eq. (3) simplifies to

$$
\left.D_{e f f} \frac{\partial c_{p}}{\partial r}\right|_{r=R_{p}}=k_{e x t}\left(c-\left.c_{p}\right|_{r=R_{p}}\right) .
$$

Moreover, appropriate inlet and outlet boundary conditions (BCs) are required for Eqs. (1) and (2).

The following two types of boundary conditions are considered for Eq. (1).

\section{Boundary conditions of type I: Robin (or Danckwerts) type inlet BCs}

In this case, the Robin type boundary condition, known in chemical engineering as Danckwerts boundary condition, is applied at the column inlet (e.g. Danckwerts (1953))

$$
-\frac{D_{L}}{u} \frac{\partial c}{\partial z}+\left.c\right|_{z=0}=\left\{\begin{array}{lc}
c_{i n j}, & \text { if } 0<t \leq t_{i n j} \\
0, & t>t_{i n j}
\end{array}\right.
$$

where $c_{i n j}$ denotes the concentration of the solute in the injected sample and $t_{i n j}$ is the time of injection. At the outlet of the column of finite length $L$, the following Neumann outflow boundary condition is used:

$$
\frac{\partial c(L, t)}{\partial z}=0
$$

\section{Boundary conditions of type II: Dirichlet inlet BCs}

Alternatively, the simpler Dirichlet boundary conditions was considered at the column inlet

$$
\left.c\right|_{z=0}=\left\{\begin{array}{lr}
c_{\mathrm{inj}}, & \text { if } 0<t \leq t_{\mathrm{inj}} \\
0, & t>t_{\mathrm{inj}}
\end{array}\right.
$$


together with a Neumann boundary condition for a column of hypothetically infinite length, $L=\infty$

$$
\frac{\partial c(t, \infty)}{\partial z}=0
$$

For sufficiently small dispersion coefficient, for example $D_{L} \leq 10^{-5} \mathrm{~m}^{2} / \mathrm{s}$, this Dirichlet inlet boundary condition is well applicable.

The natural boundary condition for Eqs. (2) and (9) at the center of pore is

$$
\left.\frac{\partial c_{p}}{\partial r}\right|_{r=0}=0
$$

The full analytical solution of this linear GRM is not possible, but analytical expressions for moments can be derived from the achievable solution of GRM in the Laplace domain. Two famous limiting models of GRM are the lumped kinetic model (LKM) and the equilibrium dispersive model (EDM), see Guiochon and Lin (2003); Guiochon et al. (2006).

The LKM can be obtained by simplifying the description of the mass transfer processes. The model lumps contributions of internal and external mass transport resistances quantified by $k_{e x t}$ and $D_{\text {eff }}$ into a single mass transfer coefficient $k_{L K M}$. The mass balance law of the LKM is expressed as

$$
\begin{aligned}
\frac{\partial c}{\partial t}+u \frac{\partial c}{\partial z} & =D \frac{\partial^{2} c}{\partial z^{2}}-\frac{k_{L K M}}{\epsilon}\left(q^{*}-q\right) \\
\frac{\partial q}{\partial t} & =\frac{k_{L K M}}{1-\epsilon}\left(q^{*}-q\right) \\
q^{*} & =a c
\end{aligned}
$$

The EDM assumes that all mass transfer kinetics are of infinite rate, i.e. $k_{\text {ext }} \rightarrow \infty$, $D_{s} \rightarrow \infty$ and $D_{p} \rightarrow \infty$. Analytical solutions and a moment analysis for the LKM and 
EDM were recently presented in Javeed et al. (2013). In this work, relationships are derived between the kinetic parameters of GRM and LKM in order to match their first four moments (see Appendix C).

\section{Analytical Solutions of GRM for Linear Isotherms}

In this section, solutions of linear GRM are presented for Danckwerts (Eq. (12a)) and Dirichlet (Eq. (13a)) inlet boundary conditions. The GRM can conveniently be solved by means of Laplace transformation. The Laplace transformation is defined as

$$
\bar{c}(s, z)=\int_{0}^{\infty} \mathrm{e}^{-\mathrm{st}} \mathrm{c}(\mathrm{t}, \mathrm{z}) \mathrm{dt}, \quad \mathrm{t} \geq 0 .
$$

By applying the above Laplace definition to the model Eq. (1), we obtain

$$
s \bar{c}+u \frac{d \bar{c}}{d z}=D_{L} \frac{d^{2} \bar{c}}{d z^{2}}-\frac{3}{R_{p}} F k_{e x t}\left(\bar{c}-\left.\bar{c}_{p}\right|_{r=R_{p}}\right) .
$$

While, the Laplace transformation of Eq. (9) is given as

$$
a^{*} s \bar{c}_{p}=D_{e f f}\left(\frac{d^{2} \bar{c}_{p}}{d r^{2}}+\frac{2}{r} \frac{d \bar{c}_{p}}{d r}\right)
$$

The general solution of Eq. (18) is given as (see Appendix A)

$$
\bar{c}_{p}(s, z, r)=\frac{1}{r}\left[\frac{d_{1}}{\sqrt{\alpha(s)}} \sinh (\sqrt{\alpha(s)} r)+d_{2} \cosh (\sqrt{\alpha(s)} r)\right] .
$$

By using the boundary conditions (9) and (14), Eq. (19) gives

$$
d_{1}=\frac{B_{p} \bar{c} / \sinh \left(\sqrt{\alpha(s)} R_{p}\right)}{\left(B_{p}-1\right) / \sqrt{\alpha(s)} R_{p}+\operatorname{coth}\left(\sqrt{\alpha(s)} R_{p}\right)}, \quad d_{2}=0 .
$$

At $r=R_{p}$, Eqs. (19) and (20) gives

$$
\left.\bar{c}_{p}\right|_{r=R_{p}}=\bar{c} f(s)
$$


where

$$
f(s)=\frac{B_{p}}{B_{p}-1+\sqrt{\alpha(s)} R_{p} \operatorname{coth}\left(\sqrt{\alpha(s)} R_{p}\right)}, \quad B_{p}=\frac{k_{e x t} R_{p}}{D_{e f f}}, \quad \alpha(s)=\frac{a^{*} s}{D_{e f f}} .
$$

Introducing Eqs. (21) in Eq. (17), we get the following ordinary differential equation

$$
\phi(s) \bar{c}+u \frac{d \bar{c}}{d z}=D_{L} \frac{d^{2} \bar{c}}{d z^{2}}
$$

where

$$
\phi(s)=s+k_{e x t} \frac{3}{R_{p}} F(1-f(s)) .
$$

The solution of this equation is given as

$$
\bar{c}(s, z)=A e^{\lambda_{1} z}+B e^{\lambda_{2} z}
$$

where

$$
\lambda_{1,2}=\frac{u}{2 D_{L}}\left(1 \mp \sqrt{1+\frac{4 D_{L} \phi(s)}{u^{2}}}\right) .
$$

The boundary conditions considered above can be used to obtain the values of integration constants $A$ and $B$ in Eq. (25).

\section{Boundary conditions of type I: Robin (or Danckwerts) type BCs}

First, the boundary conditions in Eqs. (12a) and (12b) are considered to obtain the values

of $\mathrm{A}$ and $\mathrm{B}$ in Eq. (25). The inlet Danckwerts boundary condition in Eq. (12a) can be rewritten as

$$
-\frac{1}{v} \frac{\partial c}{\partial z}+\left.c(t, z)\right|_{z=0}=\left\{\begin{array}{lr}
c_{\mathrm{inj}}, & 0<t \leq t_{\mathrm{inj}} \\
0, & t>t_{\mathrm{inj}}
\end{array}\right.
$$


It has the Laplace transformation

$$
\bar{c}(s, 0)=\frac{c_{\mathrm{inj}}}{s}\left(1-e^{-s t_{\mathrm{inj}}}\right)+\left.\frac{1}{v} \frac{d \bar{c}}{d x}\right|_{z=0},
$$

where

$$
v=\frac{u}{D_{L}}
$$

Similarly, the Laplace transformation of Eq. (12b) is given as

$$
\left.\frac{d \bar{c}(s, z)}{d z}\right|_{z=L}=0 \text {. }
$$

Thus, the values of $A$ and $B$ have the following forms

$$
\begin{gathered}
A=\frac{c_{\text {inj }}\left(1-e^{-s t_{\text {inj }}}\right)}{s} \frac{\lambda_{2} \exp \left(\lambda_{2} L\right)}{\left(1-\frac{\lambda_{1}}{v}\right) \lambda_{2} \exp \left(\lambda_{2} L\right)-\left(1-\frac{\lambda_{2}}{v}\right) \lambda_{1} \exp \left(\lambda_{1} L\right)}, \\
B=-\frac{c_{\text {inj }}\left(1-e^{-s t_{\text {inj }}}\right)}{s} \frac{\lambda_{1} \exp \left(\lambda_{1} L\right)}{\left(1-\frac{\lambda_{1}}{v}\right) \lambda_{2} \exp \left(\lambda_{2} L\right)-\left(1-\frac{\lambda_{2}}{v}\right) \lambda_{1} \exp \left(\lambda_{1} L\right)} .
\end{gathered}
$$

When $t_{\text {inj }} \rightarrow \infty$, the injection causes just a breakthrough curve. In such a situation, the values of $A$ and $B$ in Eqs. (31)-(32) reduce to

$$
\begin{gathered}
A=\frac{c_{\text {inj }}}{s} \frac{\lambda_{2} \exp \left(\lambda_{2} L\right)}{\left(1-\frac{\lambda_{1}}{v}\right) \lambda_{2} \exp \left(\lambda_{2} L\right)-\left(1-\frac{\lambda_{2}}{v}\right) \lambda_{1} \exp \left(\lambda_{1} L\right)}, \\
B=-\frac{c_{\text {inj }}}{s} \frac{\lambda_{1} \exp \left(\lambda_{1} L\right)}{\left(1-\frac{\lambda_{1}}{v}\right) \lambda_{2} \exp \left(\lambda_{2} L\right)-\left(1-\frac{\lambda_{2}}{v}\right) \lambda_{1} \exp \left(\lambda_{1} L\right)} .
\end{gathered}
$$

The complete solutions for pulse responses and breakthrough curves are given by Eq. (25) together with Eqs. (31)-(32) and Eqs. (33)-(34), respectively.

\section{Boundary conditions of type II: Dirichlet BCs}

Now, we consider the boundary conditions given by Eqs. (13a) and (13b). Their Laplace transformations are given as

$$
\bar{c}(s, 0)=\frac{c_{\mathrm{inj}}\left(1-e^{-s t_{\mathrm{inj}}}\right)}{s}, \quad \frac{d \bar{c}}{d z}(s, \infty)=0 .
$$


Thus, the values of $A$ and $B$ take the following forms

$$
A=\frac{c_{\mathrm{inj}}\left(1-e^{-s t_{\mathrm{inj}}}\right)}{s}, \quad B=0
$$

Using the values of $A$ and $B$ in Eq. (25), we get

$$
\bar{c}(s, z)=\frac{c_{\mathrm{inj}}\left(1-e^{-s t_{\mathrm{inj}}}\right)}{s} \exp \left[\frac{u z}{2 D_{L}}\left(1-\sqrt{1+\frac{4 D_{L} \phi(s)}{u^{2}}}\right)\right] .
$$

When $t_{\text {inj }} \rightarrow \infty$, the injection causes again a complete breakthrough curve. For this case, the solution in Eq. (37) reduce to the following form

$$
\bar{c}(s, z)=\frac{c_{\mathrm{inj}}}{s} \exp \left[\frac{u z}{2 D_{L}}\left(1-\sqrt{1+\frac{4 D_{L} \phi(s)}{u^{2}}}\right)\right] .
$$

This completes the discussion of analytical solutions for the single component linear GRM in the Laplace domain.

There is no possibility to analytically transform back the Laplace domain solution in time domain. Numerical Laplace inversion can be applied to obtain a discrete solution in time. In this technique, the integral of inverse Laplace transformation is approximated by Fourier series, see for example Rice et al. (1995). However, this solution is not helpful to study the behavior of chromatographic bands in the column. For that reason, an analysis of the moments is presented in the next section to serve this purpose.

\section{Moments of the General Rate Model}

Moment analysis is an effective method for deducing important information about the retention and mass transfer processes in chromatographic columns, see e.g. Guiochon et al. (2006); Kucera (1965); Miyabe et al. (2007, 2009); Ruthven (1984); Schneider and Smith 
(1968) as well as Suzuki et al. (1971). The Laplace transformation can be used as a basic tool to obtain moments. The retention equilibrium-constant and parameters of the mass transfer kinetics in the column are related to the moments in the Laplace domain. In this section, the description of chromatographic peaks by means of statistical moments is presented. The central moments up to fourth order for the GRM are calculated for different sets of BCs. In order to calculate analytical moments for rectangular concentration pulses of finite width, the following moment generating property of the Laplace transform is exploited (e.g. Van der Laan (1958))

$$
\mu_{0}=\lim _{s \rightarrow 0}(\bar{c}(s, z=L)), \quad \mu_{n}=(-1)^{n} \frac{1}{\mu_{0}} \lim _{s \rightarrow 0} \frac{d^{n}(\bar{c}(s, z=L))}{d s^{n}}, \quad n=1,2,3, \cdots .
$$

For the case of continuous injection $(t \rightarrow \infty)$, the above formulas need to be modified to generate closed responses:

$$
\mu_{0}=\lim _{s \rightarrow 0}(s \bar{c}(s, z=L)), \quad \mu_{n}=(-1)^{n} \frac{1}{\mu_{0}} \lim _{s \rightarrow 0} \frac{d^{n}(s \bar{c}(s, z=L))}{d s^{n}}, \quad n=1,2,3, \cdots .
$$

In this manuscript, the first four moments for GRM related to injected rectangular concentrations profiles (finite feed volumes) are calculated. Appendix B presents the complete derivation of the moments and Table 1 summarizes the moment results for the two sets of BCs. It is well known that the first moment $\mu_{1}$ corresponds to the retention time $t_{R}$. The value of the equilibrium constant $a$ can be estimated from the slopes of a straight lines, $\mu_{1}=t_{R}$ over $1 / u$ for constant column length and porosity. The effects of longitudinal diffusion are not significant with respect to retention time or first moment. The second central moment $\mu_{2}^{\prime}$ i.e. the variance of the elution profile provides information about the rates of the mass transfer processes in the column. The third central moment $\mu_{3}^{\prime}$ quantifies 
the front asymmetries. Finally, the considered fourth central moment $\mu_{4}^{\prime}$ measures the kurtosis. Kurtosis determines the "peakedness" that describes the shape of a probability distribution. A comparison of analytical moments based on solutions in the Laplace domain and numerically calculated moments is provided below for various test problems. Furthermore, a comparison of the derived moment expressions with the recently presented model of the LKM in Javeed et al. (2013) is given.

\section{Numerical Test Problems}

In this section, the analytical solutions presented above are validated by considering several test problems. A second-order accurate finite volume scheme (FVS) of Koren was chosen to solve Eqs. (1)-(14) for verifying the analytical results. For complete derivation of this numerical method, readers are referred to the article by Javeed et al. (2011). All parameters used in the test problems are given in Table 2.

\section{Comparison of analytical and numerical solutions}

In this part, analytical and numerical solutions are compared by considering the two pairs of boundary conditions (BCs), given by Eqs. (12a)-(12b) and Eqs. (13a)-(13b). In Figure 1, a rectangular pulse of finite width is injected and described by the Danckwerts inlet boundary condition (12a) considering for illustration two velocities $u=0.3 \mathrm{~cm} / \mathrm{min}$ and $u=0.6 \mathrm{~cm} / \mathrm{min}$. The figure compares the solutions of numerical inversion of the Laplace domain solution (Eq. (25) together with Eqs. (31) and (32)) in the time domain and FVS. Figure 2 shows the comparison of solutions for continuously injected concentrations. Good 
agreements of the obtained concentration profiles verify the accuracy of numerical Laplace inversion and FVS.

In Figures 3 and 4 results are shown for rectangular pulses of finite and infinite widths injected as Dirichlet inlet BCs. Good agreement of the solution profiles demonstrates again the consistency and accuracy of both methods. Numerical Laplace inversion is a suitable method when analytical Laplace inversion of the solution from Laplace domain is not possible.

\section{Effect of boundary conditions}

The results shown in Figure 5 illustrate the importance of using the more accurate Danckwerts BCs when the Peclet numbers are relatively small, e.g. $P e \leq 10$. Hereby the Peclet number is defined as

$$
P e=\frac{L u}{D_{L}},
$$

where $L$ denotes the column length. For such large effects of dispersion, visible differences can be observed between the results for Dirichlet and Danckwerts BCs. On the other hand, for larger values of Peclet number $(P e>>10)$ or smaller effects of axial dispersion, solutions with Dirichlet and Danckwerts boundary conditions are analogous.

In the following calculations, only Danckwerts boundary conditions were considered.

\section{Effects of axial dispersion and diffusion}

As the effect of dispersion via the $P e$ number (Eq. (41)), the effect of intraparticle diffusion can be quantified in a dimensionless way using the following Biot number $B i$ which 
quantifies the ratio of diffusion and convection time constants:

$$
B i=\frac{D_{e f f} / R_{p}^{2}}{u / L}
$$

Figure 6 (left) shows the effect of Peclet number by considering different values of $P e$ (or $D_{L}$ ) using $u=0.3 \mathrm{~cm} / \mathrm{min}$ and keeping $B i=1$ fixed. It demonstrates that for this Biot number in the range considered relative small differences are observed by significantly varying the values of $P e\left(\right.$ or $\left.D_{L}\right)$.

Figure 6 (right), shows the effects of $B_{i}$ (or $D_{\text {eff }}$ ) using $u=0.3 \mathrm{~cm} / \mathrm{min}$ for a fixed $P e=100$. This figure shows that larger values of $B i$ sharpen the concentration profiles.

\section{Discussion on analytically and numerically determined moments}

This part presents a comparison and analysis of analytically and numerically determined temporal moments of the GRM. Appendix B presents the first four moments for considered BCs. The numerical moments were obtained by integrating profiles generated with the high resolution FVS, see Javeed et al. (2011).

The normalized $n$-th temporal moment of the band profile at the outlet of a column of length $z=L$ is given as

$$
\mu_{n}=\frac{\int_{0}^{\infty} c(t, z=L) t^{n} d t}{\int_{0}^{\infty} c(t, z=L) d t}
$$

While, the corresponding $n$-th central moment is expressed as

$$
\mu_{n}^{\prime}=\frac{\int_{0}^{\infty} c(t, z=L)\left(t-\mu_{1}\right)^{n} d t}{\int_{0}^{\infty} c(t, z=L) d t}
$$

The trapezoidal rule is applied to approximate the integrals in Eqs. (43) and (44). For continuous injections $\left(t_{\mathrm{inj}} \rightarrow \infty\right)$ derivatives of the concentration profiles were used to 
calculate the moments of the transformed step responses, see Javeed et al. (2013). Eqs. (43) and (44) were also used to evaluate the solutions obtained by numerical inversion of the analytical Laplace domain solutions.

A quantitative comparison of analytical, numerical Laplace inversion and FVS moments is presented in Figure 7 over different powers of the inverse flow rates considering Danckwerts BCs. The first moments $\mu_{1}$ reveal the expected linear trends over $1 / u$, c.f. Figure 7 (top: left). Figure 7 (top: right) displays the second central moments $\mu_{2}^{\prime}$ that quantifies the variance. The third central moments $\mu_{3}^{\prime}$ that describes the skewness of the profiles is shown in Figure 7 (bottom: left). The fourth central moments $\mu_{4}^{\prime}$ is related to kurtosis and is presented in Figure 7 (bottom: right). A similar quantitative comparison of analytical and numerical moments over different inverse flow rates considering Dirichlet BCs is shown in Figure 8. Good agreements of results demonstrate the correctness of analytical calculations and high precision of FVS. It is well known that these moments can be used to calculate the frequently applied number of theoretical plates $N$ (e.g. Guiochon and Lin (2003))

$$
N=\frac{\left(\mu_{1}^{\prime}\right)^{2}}{\mu_{2}^{\prime}} .
$$

The skewness is a measure of the degree of asymmetry of a distribution. It can be evaluated using

$$
\beta=\frac{\mu_{3}^{\prime}}{\left(\mu_{2}^{\prime}\right)^{3 / 2}} .
$$

The skewness for a normal distribution is zero, and any symmetric data should have a skewness near zero. Negative values of the skewness indicate that data are left skewed and positive values indicate the right skewed data. 
For illustration of the skewness, concentration profiles considering different velocities $u$ are displayed in Figure 9 using $D_{L}=0.002$ and $D_{\text {eff }}=1.0^{-6} \mathrm{~cm}^{2} / \mathrm{min}$. For a small velocity $u=0.3 \mathrm{~cm} / \mathrm{min}$, the value for the skewness is $\beta=0.6411$, while for $u=0.9 \mathrm{~cm} / \mathrm{min}$ holds $\beta=0.5612$. With these values of $\beta$ one can predict that the concentration profile is more asymmetrical for $u=0.3 \mathrm{~cm} / \mathrm{min}$ as compared to $u=0.9 \mathrm{~cm} / \mathrm{min}$ which is clearly depicted in Figure 9.

The fourth central moment, i.e. kurtosis, measures the profiles peakedness or flatness relative to a normal distribution. In general, the kurtosis is a descriptor of the shape of a probability distribution. It is instructive to use an adjusted version of Pearson's kurtosis, the excess kurtosis, see DeCarlo (1997). The excess kurtosis compares the shape of a given distribution to that of the normal distribution. Distributions with negative or positive excess kurtosis are called platykurtic distributions or leptokurtic distributions, respectively. The following definition quantifies the excess kurtosis

$$
\gamma=\frac{\mu_{4}^{\prime}}{\left(\mu_{2}^{\prime}\right)^{2}}-3 .
$$

A high kurtosis distribution has a sharper peak and a broader tails than the normal distribution, while a low kurtosis distribution has a more rounded peak and thinner tails. Distributions with zero excess kurtosis, as the normal distribution, are called mesokurtic. The fourth central moment associated with kurtosis is used to study the flatness of chromatogram elution. Figure 10, displays the effects of different velocities $u$ on kurtosis using $D_{\text {eff }}=6.3835 \times 10^{-5} \mathrm{~cm}^{2} / \mathrm{min}$. For a relatively small value of velocity, for instance $u=0.1 \mathrm{~cm} / \mathrm{min}$, the excess kurtosis value is $\gamma=0.1679$. This depicts a tendency towards 
the normal distribution. For a larger velocity $u=5 \mathrm{~cm} / \mathrm{min}$, the value of the excess kurtosis is $\gamma=-1.1279$. This predicts that the distribution is more uniform which corresponds more to the rectangular injection profiles of finite widths.

In this work, also relationships were derived to reveal the connection between kinetic parameters of the linear GRM and LKM and to match the first four moments for Danckwerts BCs. These relations, given in appendix $\mathrm{C}$ as Eqs. (C-1), (C-2), (C-3) and (C-6), can be used to match the first four moments of LKM with those of GRM. Moreover, also the moments of EDM can be obtained by putting $k_{L K M} \rightarrow \infty$ or $k_{e x t} \rightarrow \infty$ and $D_{\text {eff }} \rightarrow \infty$ in Eqs. (C-2), (C-3) and (C-6).

Figure 11 shows the moments of GRM and LKM after matching them through relations given by Eqs. (C-2), (C-3) or (C-6). It demonstrates that both models produce same second moments if $K_{L K M}$ in Eq. (C-2) is used. The third moments are identical when $K_{L K M}$ is obtained from Eq. (C-3). In addition, the fourth moments of both models are identical when $K_{L K M}$ is calculated from Eq. (C-6), see Figure 11.

For $a^{*}=a=2.0, u=0.3 \mathrm{~m} / \mathrm{s}, D_{L}=0.002 \mathrm{~cm}^{2} / \mathrm{min}$ and $D_{\text {eff }}=10^{-6} \mathrm{~cm}^{2} / \mathrm{min}$, one obtain $k_{L K M}=0.25 \mathrm{~min}^{-1}, k_{L K M}=0.217 \mathrm{~min}^{-1}$ and $k_{L K M}=0.235 \mathrm{~min}^{-1}$ from Eqs. (C-2), (C-3) and (C-6), respectively. Table 3, shows the values of the moments of LKM and GRM. It can be observed from the table that both models have identical values of $\mu_{2}^{\prime}$ when the value of $K_{L K M}$ was obtained from Eq. (C-2), while $\mu_{3}^{\prime}$ and $\mu_{4}^{\prime}$ are different by $24 \%$ and $10 \%$, respectively. Secondly, both models have the same values of $\mu_{3}^{\prime}$ when $K_{L K M}$ was obtained from Eq. (C-3) but $\mu_{2}^{\prime}$ and $\mu_{4}^{\prime}$ differ by $10 \%$ and $14 \%$, respectively. Finally, when $K_{L K M}$ was measured by using Eq. (C-6), both models have the same $\mu_{4}^{\prime}$ but different 
$\mu_{2}^{\prime}$ and $\mu_{3}^{\prime}$ by approximately $4 \%$ and $14 \%$. It can be noticed that the value of $K_{L K M}$ from Eq. (C-6) has produced less error in $\mu_{2}^{\prime}$ as compared to the value of $K_{L K M}$ from Eq. (C-3). Finally, Figure 12 shows the comparison of concentration profiles from GRM and LKM for the aforementioned three different values of $k_{L K M}$. Small difference can be observed in the concentration profiles of LKM for these values of $k_{L K M}$. For $k_{L K M}=0.25 \mathrm{~min}^{-1}$, the variance of concentration profile from LKM is identical to the variance of concentration profile from GRM. For other values of $k_{L K M}$, the variances of concentration profiles from GRM and LKM deviate from each other as observed in Table 3.

\section{Conclusion}

Analytical solutions and moments of single component linear GRM were presented for two sets of boundary conditions. The Laplace transformation was used as a basic tool to obtain solutions in the Laplace domain. Due to the fact that these solutions could not be inverted back analytically, numerical inversion was used to get concentration profiles in the time domain. Analytical expression of the first four temporal moments were derived from the solutions in Laplace domain. The moments were used to analyze the retention times, band broadenings, front asymmetries and kurtosis of the elution profiles. The analytical results were validated against the numerical results of second order finite volume scheme. Good agreements between analytically and numerically determined results verified the correctness of analytical solutions and the accuracy of suggested numerical scheme. Relationships were derived among the kinetic parameters of GRM and LKM to match their first four moments. These relations can be used to estimate parameters of the simple LKM from 
GRM parameters.

Work is in progress to utilize the analytically determined moments for parameters estimation.

\section{Appendix A}

Derivation of series solution of Eq. (18)

Here, the series solution of Eq. (18) is presented, the equation can be rewritten as

$$
r \frac{d^{2} \overline{c_{p}}}{d r^{2}}+2 \frac{d \overline{c_{p}}}{d r}=r \frac{a^{*} s}{D_{e f f}} \overline{c_{p}}
$$

Let

$$
\alpha:=\alpha(s)=\frac{a^{*} s}{D_{e f f}}
$$

Let $\overline{c_{p}}=\sum_{n=0}^{\infty} a_{n} r^{n+x}$, where $x$ is any positive integer, then Eq. (A-1) becomes

$$
\sum_{n=0}^{\infty}(n+x)(n+x-1) a_{n} r^{n+x-1}+\sum_{n=0}^{\infty} 2(n+x) a_{n} r^{n+x-1}-\sum_{n=0}^{\infty} \alpha a_{n} r^{n+x+1}=0
$$

or

$$
\sum_{n=0}^{\infty}(n+x)(n+x+1) a_{n} r^{n+x-1}-\sum_{n=0}^{\infty} \alpha a_{n} r^{n+x+1}=0
$$

Take $k=n-1$ and $k=n+1$

$r^{x}\left[a_{0}\left(x^{2}+x\right) r^{-1}+a_{1}(x+1)(x+2) r+\sum_{k=1}^{\infty}\left(a_{k+1}(k+x+1)(k+x+2)-\alpha a_{k-1}\right) r^{k}\right]=0$. 


\section{Comparing coefficients}

$$
x^{2}+x=0 \Rightarrow x(x+1)=0 \Rightarrow x_{1}=0, x_{2}=-1, \quad x_{1}-x_{2}=1 \text { (positive integer) } .
$$

Now $a_{1}=0$, and

$$
a_{k+1}(k+x+1)(k+x+2)-\alpha a_{k-1}=0,
$$

implies

$$
a_{k+1}=\frac{\alpha a_{k-1}}{(k+x+1)(k+x+2)}, \quad k=1,2, \cdots .
$$

Now for $x=0$,

$$
a_{k+1}=\frac{\alpha a_{k-1}}{(k+1)(k+2)}
$$

$k=1$, gives

$$
a_{2}=\frac{\alpha a_{0}}{2.3}=\frac{\alpha a_{0}}{3 !}
$$

$k=2$, gives

$$
a_{3}=0,
$$

$k=3, k=4$, and $k=5$ gives

$$
a_{4}=\frac{\alpha^{2} a_{0}}{5 !}, \quad a_{5}=0, \quad a_{6}=\frac{\alpha^{3} a_{0}}{7 !} .
$$

Thus, induction gives

$$
a_{2 n-1}=0, \quad a_{2 n}=\frac{\alpha^{n} a_{0}}{(2 n+1) !}
$$


Hence, $\bar{c}=\sum_{n=0}^{\infty} a_{n} r^{n+x}$ for $x=0$ gives

$$
\overline{c_{p}}=\sum_{n=0}^{\infty} \frac{\alpha^{n} a_{0}}{(2 n+1) !} r^{2 n} .
$$

Similarly, for $x=-1$ we can obtain

$$
\overline{c_{p}}=\sum_{n=0}^{\infty} \frac{\alpha^{n} a_{0}}{(2 n) !} r^{2 n-1} .
$$

Thus, the combined solution can be written as

$$
\overline{c_{p}}=d_{1} \sum_{n=0}^{\infty} \frac{\alpha^{n}}{(2 n+1) !} r^{2 n}+d_{2} \sum_{n=1}^{\infty} \frac{\alpha^{n}}{(2 n) !} r^{2 n}
$$

or

$$
\overline{c_{p}}=\frac{1}{r}\left[\frac{d_{1}}{\sqrt{\alpha}} \sinh (\sqrt{\alpha} r)+d_{2} \cosh (\sqrt{\alpha} r)\right],
$$

Applying the boundary conditions (11) and (14), we get

$$
d_{1}=\frac{B_{p} \bar{c} / \sinh \left(\sqrt{\alpha} R_{p}\right)}{\left(B_{p}-1\right) / \sqrt{\alpha} R_{p}+\operatorname{coth}\left(\sqrt{\alpha} R_{p}\right)}, \quad d_{2}=0
$$

Thus, the final solution in Eq. (A-17) using Eq. (A-18) takes the following form

$$
\overline{c_{p}}=\frac{R_{p}}{r}\left[\frac{\frac{B_{p}}{\sinh \left(\sqrt{\alpha(s)} R_{p}\right)} \sinh (\sqrt{\alpha(s)} r)}{B_{p}-1+\sqrt{\alpha(s)} R_{p} \operatorname{coth}\left(\sqrt{\alpha(s)} R_{p}\right)}\right] \bar{c} .
$$

At $r=R_{p}$, Eqs. (19) and (20) gives

$$
\left.\bar{c}_{p}\right|_{r=R_{p}}=\bar{c} f(s)
$$

where

$$
f(s)=\frac{B_{p}}{B_{p}-1+\sqrt{\alpha(s)} R_{p} \operatorname{coth}\left(\sqrt{\alpha(s)} R_{p}\right)}, \quad B_{p}=\frac{k_{e x t} R_{p}}{D_{e f f}}, \quad \alpha(s)=\frac{a^{*} s}{D_{e f f}} .
$$




\section{Appendix B}

Here, the complete derivations of moments are presented for GRM using two types of boundary conditions.

\section{Boundary conditions of type I: Robin (or Danckwerts) type boundary condi- tions}

Here, the boundary conditions given by Eqs. (12a) and (12b) are considered. The final solution is given by Eqs. (25), (31), and (32). The moments of this solutions are given below.

\section{Zeroth moment:}

The zeroth moment for rectangular profiles is given as

$$
\mu_{0}=c_{\mathrm{inj}} t_{\mathrm{inj}}
$$

The zeroth moment for continuous breakthrough curves is provided as

$$
\mu_{0}=c_{\text {inj }}
$$

\section{First moment:}

The first temporal moment using Eq. (39) is calculated as

$$
\mu_{1}=\frac{t_{\text {inj }}}{2}+\frac{L}{u}\left(1+a^{*} F\right)
$$

For continuous breakthrough curves, Eq. (40) can be used to obtain the following expression of first moment

$$
\mu_{1}=\frac{L}{u}\left(1+a^{*} F\right)
$$




\section{Second moment:}

The second temporal normalized moment based on Eq. (39) is given as

$$
\begin{aligned}
\mu_{2}= & \frac{t_{\mathrm{inj}}^{2}}{3}+t_{\mathrm{inj}} \frac{L}{u}\left(1+a^{*} F\right)+\frac{2 D_{L}^{2}\left(1+a^{*} F\right)^{2}}{u^{4}}\left(-1+\frac{L u}{D_{L}}+\frac{L^{2} u^{2}}{2 D_{L}^{2}}+e^{-\frac{L u}{D_{L}}}\right) \\
& +\frac{L F}{u}\left(\frac{2 R_{p} a^{* 2}}{3 k_{\text {ext }}}+\frac{2 R_{p}^{2} a^{* 2}}{15 D_{\text {eff }}}\right) .
\end{aligned}
$$

The second central moment for rectangular profiles is expressed as

$$
\mu_{2}^{\prime}=\mu_{2}-\mu_{1}^{2}=\frac{t_{\mathrm{inj}}^{2}}{12}+\frac{2 L D_{L}\left(1+a^{*} F\right)^{2}}{u^{3}}\left(1+\frac{D_{L}}{L u}\left(e^{-\frac{L u}{D_{L}}}-1\right)\right)+\frac{L F}{u}\left(\frac{2 R_{p} a^{* 2}}{3 k_{e x t}}+\frac{2 R_{p}^{2} a^{* 2}}{15 D_{\text {eff }}}\right) .
$$

For continuous breakthrough curves, the second central moment is obtained using Eq. (40)

$$
\mu_{2}^{\prime}=\frac{2 L D_{L}\left(1+a^{*} F\right)^{2}}{u^{3}}\left[1+\frac{D}{L u}\left(e^{-\frac{L u}{D_{L}}}-1\right)\right]+\frac{L F}{u}\left(\frac{2 R_{p} a^{* 2}}{3 k_{e x t}}+\frac{2 R_{p}^{2} a^{* 2}}{15 D_{e f f}}\right)
$$

\section{Third moment:}

The third temporal normalized moment exploiting Eq. (39) is provided as

$$
\begin{aligned}
\mu_{3}= & \frac{\left(1+a^{*} F\right)^{3}}{u^{6}}\left(L^{3} u^{3}+6 D_{L} L^{2} u^{2}+6 D_{L}^{2} L u-24 D_{L}^{3}+18 D_{L}^{2} L u e^{-\frac{L u}{D_{L}}}+24 D_{L}^{3} e^{-\frac{L u}{D_{L}}}\right) \\
& +\frac{6 L D_{L} F\left(1+a^{*} F\right)}{u^{3}}\left(\frac{2 R_{p} a^{* 2}}{3 k_{e x t}}+\frac{2 R_{p}^{2} a^{* 2}}{15 D_{\text {eff }}}\right)\left[\frac{D_{L}}{L u}\left(e^{-\frac{L u}{D_{L}}}-1\right)+\left(1+\frac{L u}{2 D_{L}}\right)\right] \\
& +t_{\mathrm{inj}} \frac{3 L a^{* 2} F}{u}\left(\frac{R_{p}}{3 k_{e x t}}+\frac{R_{p}^{2}}{15 D_{\text {eff }}}\right)+\frac{L a^{* 3} F}{u}\left(\frac{4 R_{p}^{4}}{105 D_{\text {eff }}^{2}}+\frac{4 R_{p}^{3}}{15 k_{\text {ext }} D_{\text {eff }}}+\frac{2 R_{p}^{2}}{3 k_{\text {ext }}^{2}}\right) \\
& +\frac{t_{\mathrm{inj}}^{3}}{4}+t_{\mathrm{inj}}^{2} \frac{L}{u}\left(1+a^{*} F\right)+3 t_{\mathrm{inj}}(1+\epsilon F)^{2} \frac{L D_{L}}{u^{3}}\left[\frac{D_{L}}{L u}\left(e^{-\frac{L u}{D_{L}}}-1\right)+1+\frac{L u}{2 D_{L}}\right] .
\end{aligned}
$$


The third central moment formula is given as

$$
\begin{aligned}
\mu_{3}^{\prime}= & \mu_{3}-3 \mu_{1} \mu_{2}+2 \mu_{1}^{3} \\
= & \frac{12 L D_{L}^{2}\left(1+a^{*} F\right)^{3}}{u^{5}}\left[\left(1+\frac{2 D_{L}}{L u}\right) e^{-\frac{L u}{D_{L}}}+\left(1-\frac{2 D_{L}}{L u}\right)\right] \\
& +\frac{6 L D_{L} F\left(1+a^{*} F\right)}{u^{3}}\left(\frac{2 R_{p} a^{* 2}}{3 k_{\text {ext }}}+\frac{2 R_{p}^{2} a^{* 2}}{15 D_{\text {eff }}}\right)\left[\frac{D_{L}}{L u}\left(e^{-\frac{L u}{D_{L}}}-1\right)+1\right] \\
& +\frac{L a^{* 3} F}{u}\left(\frac{4 R_{p}^{4}}{105 D_{\text {eff }}^{2}}+\frac{4 R_{p}^{3}}{15 k_{\text {ext }} D_{\text {eff }}}+\frac{2 R_{p}^{2}}{3 k_{\text {ext }}^{2}}\right) .
\end{aligned}
$$

The third central moment is same for continuous breakthrough curve. 


\section{Fourth moment:}

The fourth moment can be obtained using Eq. (39) as

$$
\begin{aligned}
& \mu_{4}=\frac{D_{L}^{4}\left(1+a^{*} F\right)^{4}}{u^{8}}\left[24 e^{-\frac{2 L u}{D_{L}}}+\left(312+108 \frac{L^{2} u^{2}}{D_{L}^{2}}+360 \frac{L u}{D_{L}}\right) e^{-\frac{L u}{D_{L}}}+\frac{L^{4} u^{4}}{D_{L}^{4}}+12 \frac{L^{3} u^{3}}{D_{L}^{3}}\right. \\
& \left.+48 \frac{L^{2} u^{2}}{D_{L}^{2}}-336\right]+\frac{12 D_{L}^{3}\left(1+a^{*} F\right)^{2} F}{u^{6}}\left(\frac{R_{p} a^{* 2}}{3 k_{e x t}}+\frac{R_{p}^{2} a^{* 2}}{15 D_{\text {eff }}}\right) \\
& \cdot\left[\frac{L^{3} u^{3}}{D_{L}^{3}}+6 \frac{L^{2} u^{2}}{D_{L}^{2}}+6 \frac{L u}{D_{L}}-24+\left(24+18 \frac{L u}{D_{L}}\right) e^{-\frac{L u}{D_{L}}}\right] \\
& +\frac{4 L D_{L} F a^{* 3}(2+3 \epsilon F)}{u^{3}}\left(\frac{4 R_{p}^{4}}{105 D_{\text {eff }}^{2}}+\frac{4 R_{p}^{3}}{15 k_{\text {ext }} D_{\text {eff }}}+\frac{2 R_{p}^{2}}{3 k_{\text {ext }}^{2}}\right)\left[1-\frac{D_{L}}{L u}\left(1-e^{-\frac{L u}{D_{L}}}\right)+\frac{L u}{D_{L}}\right] \\
& +\frac{8 L F a^{* 4}}{u}\left(\frac{1}{525} \frac{R_{p}^{6}}{D_{\text {eff }}^{3}}+\frac{3}{175} \frac{R_{p}^{5}}{D_{\text {eff }}^{2} k_{\text {ext }}}+\frac{1}{15} \frac{R_{p}^{4}}{D_{\text {eff }} k_{\text {ext }}^{2}}+\frac{1}{9} \frac{R_{p}^{3}}{k_{\text {ext }}^{3}}\right) \\
& -\frac{4}{175} \frac{L F^{2} D_{L} a^{* 4} R_{p}^{4}}{u^{3} D_{\text {eff }}^{2}}\left(2-\frac{2 D_{L}}{L u}\left(1-e^{-\frac{L u}{D_{L}}}\right)+\frac{L u}{D_{L}}\right)+\frac{t_{i n j}^{4}}{5}+t_{i n j}^{3} \frac{L}{u}\left(1+a^{*} F\right) \\
& +2 t_{i n j}^{2} \frac{L F}{u}\left(\frac{2 R_{p} a^{* 2}}{3 k_{e x t}}+\frac{2 R_{p}^{2} a^{* 2}}{15 D_{e f f}}\right)+2 t_{i n j}^{2} \frac{L D_{L}}{u^{3}}\left(1+a^{*} F\right)^{2}\left[2-\frac{2 D_{L}}{L u}\left(1-e^{-\frac{L u}{D_{L}}}\right)+\frac{L u}{D_{L}}\right] \\
& +t_{i n j} \frac{2 L D_{L}^{2}(1+\epsilon F)^{3}}{u^{5}}\left(24 \frac{D_{L}}{L u}\left(e^{-\frac{L u}{D_{L}}}-1\right)+6\left(1+3 e^{-\frac{L u}{D_{L}}}\right)+6 \frac{L u}{D_{L}}+\frac{L^{2} u^{2}}{D_{L}^{2}}\right) \\
& +t_{i n j} \frac{12 L D_{L} F(1+\epsilon F)}{u^{3}}\left[\frac{R_{p} a^{* 2}}{3 k_{\text {ext }}}+\frac{R_{p}^{2} a^{* 2}}{15 D_{\text {eff }}}\right]\left(2-\frac{2 D_{L}}{L u}\left(1-e^{-\frac{L u}{D_{L}}}\right)+\frac{L u}{D_{L}}\right) \\
& +t_{i n j} \frac{2 L F a^{* 3}}{u}\left(\frac{4 R_{p}^{4}}{105 D_{\text {eff }}^{2}}+\frac{4 R_{p}^{3}}{15 k_{\text {ext }} D_{\text {eff }}}+\frac{2 R_{p}^{2}}{3 k_{\text {ext }}^{2}}\right) \text {. }
\end{aligned}
$$


The fourth central moment for rectangular profiles is calculated as:

$$
\begin{aligned}
\mu_{4}^{\prime}= & \mu_{4}-4 \mu_{1} \mu_{3}+6 \mu_{1}^{2} \mu_{2}-3 \mu_{1}^{4} \\
= & \frac{12 D_{L}^{4}\left(1+a^{*} F\right)^{4}}{u^{8}}\left[2 e^{-\frac{2 L u}{D_{L}}}+\left(4 \frac{L^{2} u^{2}}{D_{L}^{2}}+22 \frac{L u}{D_{L}}+26\right) e^{-\frac{L u}{D_{L}}}+\frac{L^{2} u^{2}}{D_{L}^{2}}+8 \frac{L u}{D_{L}}-28\right] \\
& +\frac{24 D_{L}^{3}\left(1+a^{*} F\right)^{2} F}{u^{6}}\left(\frac{R_{p} a^{* 2}}{3 k_{e x t}}+\frac{R_{p}^{2} a^{* 2}}{15 D_{\text {eff }}}\right)\left[\frac{L^{2} u^{2}}{D_{L}^{2}}+5 \frac{L u}{D_{L}}-12+\left(7 \frac{L u}{D_{L}}+12\right) e^{\left.-\frac{L u}{D_{L}}\right]}\right. \\
& +\frac{2 L D_{L} F a^{* 3}}{u^{3}}\left[\frac{4 R_{p}^{4}}{105 D_{\text {eff }}^{2}}+\frac{4 R_{p}^{3}}{15 k_{e x t} D_{\text {eff }}}+\frac{2 R_{p}^{2}}{3 k_{\text {ext }}^{2}}\right]\left[\left(4+6 a^{*} F\right)\left(1-\frac{D_{L}}{L u}\left(1-e^{-\frac{L u}{D_{L}}}\right)\right)+\frac{a^{*} F L u}{D_{L}}\right] \\
& +\frac{8 L F a^{* 4}}{u}\left(\frac{1}{525} \frac{R_{p}^{6}}{D_{e f f}^{3}}+\frac{3}{175} \frac{R_{p}^{5}}{D_{e f f}^{2} k_{e x t}}+\frac{1}{15} \frac{R_{p}^{4}}{D_{e f f} k_{e x t}^{2}}+\frac{1}{9} \frac{R_{p}^{3}}{k_{e x t}^{3}}\right) \\
& -\frac{4}{175} \frac{L F^{2} D_{L} a^{* 4} R_{p}^{4}}{u^{3} D_{e f f}^{2}}\left[2\left(1-\frac{D_{L}}{L u}\left(1-e^{-\frac{L u}{D_{L}}}\right)\right)+\frac{L u}{D_{L}}\right]+\frac{t_{\text {inj }}^{4}}{80} \\
& +t_{i n j}^{2} \frac{D_{L}^{2}(1+\epsilon F)^{2}}{u^{4}}\left[\frac{L u}{D_{L}}+e^{-\frac{L u}{D_{L}}}-1\right]+t_{i n j}^{2} \frac{L F}{u}\left[\frac{R_{p} a^{* 2}}{3 k_{e x t}}+\frac{R_{p}^{2} a^{* 2}}{15 D_{e f f}}\right] .
\end{aligned}
$$

For continuous breakthrough curves, the fourth central moment is obtained using Eq. (40):

$$
\begin{aligned}
\mu_{4}^{\prime}= & \frac{12 D_{L}^{4}\left(1+a^{*} F\right)^{4}}{u^{8}}\left[2 e^{-\frac{2 L u}{D_{L}}}+\left(4 \frac{L^{2} u^{2}}{D_{L}^{2}}+22 \frac{L u}{D_{L}}+26\right) e^{-\frac{L u}{D_{L}}}+\frac{L^{2} u^{2}}{D_{L}^{2}}+8 \frac{L u}{D_{L}}-28\right] \\
& +\frac{24 D_{L}^{3}\left(1+a^{*} F\right)^{2} F}{u_{h}^{6}}\left(\frac{R_{p} a^{* 2}}{3 k_{\text {ext }}}+\frac{R_{p}^{2} a^{* 2}}{15 D_{\text {eff }}}\right)\left[\frac{L^{2} u^{2}}{D_{L}^{2}}+5 \frac{L u}{D_{L}}-12+\left(7 \frac{L u}{D_{L}}+12\right) e^{-\frac{L u}{D_{L}}}\right] \\
& +\frac{2 L D_{L} F a^{* 3}}{u^{3}}\left[\frac{4 R_{p}^{4}}{105 D_{\text {eff }}^{2}}+\frac{4 R_{p}^{3}}{15 k_{\text {ext }} D_{\text {eff }}}+\frac{2 R_{p}^{2}}{3 k_{\text {ext }}^{2}}\right]\left[\left(4+6 a^{*} F\right)\left(1-\frac{D_{L}}{L u}\left(1-e^{-\frac{L u}{D_{L}}}\right)\right)+\frac{a^{*} F L u}{D_{L}}\right] \\
& +\frac{8 L F a^{* 4}}{u}\left(\frac{1}{525} \frac{R_{p}^{6}}{D_{\text {eff }}^{3}}+\frac{3}{175} \frac{R_{p}^{5}}{D_{\text {eff }}^{2} k_{\text {ext }}}+\frac{1}{15} \frac{R_{p}^{4}}{D_{\text {eff }} k_{\text {ext }}^{2}}+\frac{1}{9} \frac{R_{p}^{3}}{k_{\text {ext }}^{3}}\right) \\
& -\frac{4}{175} \frac{L F^{2} D_{L} a^{* 4} R_{p}^{4}}{u^{3} D_{\text {eff }}^{2}}\left[2\left(1-\frac{D_{L}}{L u}\left(1-e^{-\frac{L u}{D_{L}}}\right)\right)+\frac{L u}{D_{L}}\right] .
\end{aligned}
$$

\section{Boundary conditions of type II: Dirichlet boundary conditions}

Here, the boundary conditions given by Eqs. (13a) and (13b) are considered. The final solution is given by Eqs. (38). The moments of this solutions are given below. 


\section{Zeroth moment:}

The zeroth moment for rectangular profiles is given as

$$
\mu_{0}=\lim _{s \rightarrow 0}(\bar{c}(s, z=L))=c_{\mathrm{inj}} t_{\mathrm{inj}}
$$

The zeroth moment for continuous breakthrough curves is provided as

$$
\mu_{0}=c_{\mathrm{inj}}
$$

\section{First moment:}

The first temporal moment for rectangular profiles is calculated from Eq. (39) as

$$
\mu_{1}=\frac{t_{\mathrm{inj}}}{2}+\frac{L}{u}\left(1+a^{*} F\right)
$$

For continuous breakthrough curves, the first moment is obtained by using Eq. (40) as given below

$$
\mu_{1}=\frac{L}{u}\left(1+a^{*} F\right)
$$

\section{Second moment:}

The second temporal moment is again derived using Eq. (39) as

$\mu_{2}=\frac{t_{\mathrm{inj}}^{2}}{3}+t_{\mathrm{inj}} \frac{L}{u}\left(1+a^{*} F\right)+\frac{L^{2}}{u^{2}}\left(1+a^{*} F\right)^{2}+\frac{L F}{u}\left(\frac{2 R_{p} a^{* 2}}{3 k_{\text {ext }}}+\frac{2 R_{p}^{2} a^{* 2}}{15 D_{\text {eff }}}\right)+\frac{2 L D_{L}}{u^{3}}\left(1+a^{*} F\right)^{2}$.

Now the second central moments for rectangular profiles is given as:

$$
\mu_{2}^{\prime}=\frac{t_{\mathrm{inj}}^{2}}{12}+\frac{L F}{u}\left(\frac{2 R_{p} a^{* 2}}{3 k_{\text {ext }}}+\frac{2 R_{p}^{2} a^{* 2}}{15 D_{\text {eff }}}\right)+\frac{2 L D_{L}}{u^{3}}\left(1+a^{*} F\right)^{2}
$$


In order to obtain the second temporal moment for continuous breakthrough curves, Eq. (40) is taken into account. Then second central moment Eq. (B-18) reduces to

$$
\mu_{2}^{\prime}=\frac{L F}{u}\left(\frac{2 R_{p} a^{* 2}}{3 k_{e x t}}+\frac{2 R_{p}^{2} a^{* 2}}{15 D_{\text {eff }}}\right)+\frac{2 L D_{L}}{u^{3}}\left(1+a^{*} F\right)^{2} .
$$

\section{Third moment:}

The third temporal moment based on Eq. (39), is given as

$$
\begin{aligned}
\mu_{3}= & \frac{t_{\mathrm{inj}}^{3}}{4}+t_{\mathrm{inj}} \frac{L}{u}\left(1+a^{*} F\right)\left[t_{\mathrm{inj}}+\frac{3}{u}\left(1+a^{*} F\right)\left(\frac{D_{L}}{u}+\frac{L}{2}\right)\right]+t_{\mathrm{inj}} \frac{3 L F}{u}\left(\frac{R_{p} a^{* 2}}{3 k_{\text {ext }}}+\frac{R_{p}^{2} a^{* 2}}{15 D_{\text {eff }}}\right) \\
& +\frac{L^{3}}{u^{3}}\left(1+a^{*} F\right)^{3}\left(\frac{6 D_{L}}{L u}+\frac{12 D_{L}^{2}}{L^{2} u^{2}}+1\right)+\frac{6 L D_{L}\left(1+a^{*} F\right) F}{u^{3}}\left(\frac{2 R_{p} a^{* 2}}{3 k_{e x t}}+\frac{2 R_{p}^{2} a^{* 2}}{15 D_{\text {eff }}}\right) \\
& +\frac{L F a^{* 3}}{u}\left(\frac{4 R_{p}^{4}}{105 D_{\text {eff }}^{2}}+\frac{4 R_{p}^{3}}{15 k_{\text {ext }} D_{\text {eff }}}+\frac{2 R_{p}^{2}}{3 k_{\text {ext }}^{2}}\right)+\frac{2 L^{2}\left(1+a^{*} F\right) F}{5 u^{2}}\left(\frac{R_{p}^{2} a^{* 2}}{D_{\text {eff }}}+\frac{5 R_{p} a^{* 2}}{k_{\text {ext }}}\right) .
\end{aligned}
$$

The third central moment is calculated as:

$$
\begin{aligned}
\mu_{3}^{\prime}= & \frac{6 L D_{L}\left(1+a^{*} F\right) F}{u^{3}}\left(\frac{2 R_{p} a^{* 2}}{3 k_{e x t}}+\frac{2 R_{p}^{2} a^{* 2}}{15 D_{\text {eff }}}\right) \\
& +\frac{L F a^{* 3}}{u}\left(\frac{4 R_{p}^{4}}{105 D_{\text {eff }}^{2}}+\frac{4 R_{p}^{3}}{15 k_{\text {ext }} D_{\text {eff }}}+\frac{2 R_{p}^{2}}{3 k_{\text {ext }}^{2}}\right)+\frac{12 L D_{L}^{2}}{u^{5}}\left(1+a^{*} F\right)^{3} .
\end{aligned}
$$

The third central moment $\mu_{3}^{\prime}$ is the same for rectangular and continuous breakthrough curves. 


\section{Fourth moment:}

The fourth temporal moment is obtained as (cf. Eq. (40))

$$
\begin{aligned}
& \mu_{4}=\frac{D_{L}^{4}\left(1+a^{*} F\right)^{4}}{u^{8}}\left[24 e^{-\frac{2 L u}{D_{L}}}+\left(312+108 \frac{L^{2} u^{2}}{D_{L}^{2}}+360 \frac{L u}{D_{L}}\right) e^{-\frac{L u}{D_{L}}}+\frac{L^{4} u^{4}}{D_{L}^{4}}+12 \frac{L^{3} u^{3}}{D_{L}^{3}}\right. \\
& \left.+48 \frac{L^{2} u^{2}}{D_{L}^{2}}-336\right]+\frac{12 D_{L}^{3}\left(1+a^{*} F\right)^{2} F}{u_{h}^{6}}\left(\frac{R_{p} a^{* 2}}{3 k_{e x t}}+\frac{R_{p}^{2} a^{* 2}}{15 D_{\text {eff }}}\right) \\
& \cdot\left[\frac{L^{3} u^{3}}{D_{L}^{3}}+6 \frac{L^{2} u^{2}}{D_{L}^{2}}+6 \frac{L u}{D_{L}}-24+\left(24+18 \frac{L u}{D_{L}}\right) e^{-\frac{L u}{D_{L}}}\right] \\
& +\frac{4 L D_{L} F a^{* 3}\left(2+3 a^{*} F\right)}{u^{3}}\left(\frac{4 R_{p}^{4}}{105 D_{\text {eff }}^{2}}+\frac{4 R_{p}^{3}}{15 k_{\text {ext }} D_{\text {eff }}}+\frac{2 R_{p}^{2}}{3 k_{\text {ext }}^{2}}\right)\left[1-\frac{D_{L}}{L u}\left(1-e^{-\frac{L u}{D_{L}}}\right)+\frac{1}{2} \frac{L u}{D_{L}}\right] \\
& +\frac{8 L F a^{* 4}}{u}\left(\frac{1}{525} \frac{R_{p}^{6}}{D_{\text {eff }}^{3}}+\frac{3}{175} \frac{R_{p}^{5}}{D_{\text {eff }}^{2} k_{\text {ext }}}+\frac{1}{15} \frac{R_{p}^{4}}{D_{\text {eff }} k_{\text {ext }}^{2}}+\frac{1}{9} \frac{R_{p}^{3}}{k_{\text {ext }}^{3}}\right) \\
& -\frac{4}{175} \frac{L F^{2} D_{L} a^{* 4} R_{p}^{4}}{u^{3} D_{e f f}^{2}}\left(2+\frac{L u}{D_{L}}\right)+\frac{t_{i n j}^{4}}{5}+t_{i n j}^{3} \frac{L}{u}\left(1+a^{*} F\right) \\
& +2 t_{i n j}^{2} \frac{L F}{u}\left(\frac{2 R_{p} a^{* 2}}{3 k_{e x t}}+\frac{2 R_{p}^{2} a^{* 2}}{15 D_{e f f}}\right)+2 t_{i n j}^{2} \frac{L D_{L}}{u^{3}}\left(1+a^{*} F\right)^{2}\left[2-\frac{2 D_{L}}{L u}\left(1-e^{-\frac{L u}{D_{L}}}\right)+\frac{L u}{D_{L}}\right] \\
& +t_{i n j} \frac{2 L D_{L}^{2}\left(1+a^{*} F\right)^{3}}{u^{5}}\left(24 \frac{D_{L}}{L u}\left(e^{-\frac{L u}{D_{L}}}-1\right)+6\left(1+3 e^{-\frac{L u}{D_{L}}}\right)+6 \frac{L u}{D_{L}}+\frac{L^{2} u^{2}}{D_{L}^{2}}\right) \\
& +t_{i n j} \frac{12 L D_{L} F\left(1+a^{*} F\right)}{u^{3}}\left[\frac{R_{p} a^{* 2}}{3 k_{\text {ext }}}+\frac{R_{p}^{2} a^{* 2}}{15 D_{\text {eff }}}\right]\left(2-\frac{2 D_{L}}{L u}\left(1-e^{-\frac{L u}{D_{L}}}\right)+\frac{L u}{D_{L}}\right) \\
& +t_{i n j} \frac{2 L F a^{* 3}}{u}\left(\frac{4 R_{p}^{4}}{105 D_{e f f}^{2}}+\frac{4 R_{p}^{3}}{15 k_{\text {ext }} D_{\text {eff }}}+\frac{2 R_{p}^{2}}{3 k_{\text {ext }}^{2}}\right) \text {. }
\end{aligned}
$$


The fourth central moment for rectangular profiles is calculated as:

$$
\begin{aligned}
\mu_{4}^{\prime}= & \frac{12 D_{L}^{4}\left(1+a^{*} F\right)^{4}}{u^{8}}\left[2 e^{-\frac{2 L u}{D_{L}}}+\left(4 \frac{L^{2} u^{2}}{D_{L}^{2}}+22 \frac{L u}{D_{L}}+26\right) e^{-\frac{L u}{D_{L}}}+\frac{L^{2} u^{2}}{D_{L}^{2}}+8 \frac{L u}{D_{L}}-28\right] \\
& +\frac{24 D_{L}^{3}\left(1+a^{*} F\right)^{2} F}{u^{6}}\left(\frac{R_{p} a^{* 2}}{3 k_{e x t}}+\frac{R_{p}^{2} a^{* 2}}{15 D_{\text {eff }}}\right)\left[\frac{L^{2} u^{2}}{D_{L}^{2}}+5 \frac{L u}{D_{L}}-12+\left(7 \frac{L u}{D_{L}}+12\right) e^{-\frac{L u}{D_{L}}}\right] \\
& +\frac{2 L D_{L} F a^{* 3}}{u^{3}}\left[\frac{4 R_{p}^{4}}{105 D_{\text {eff }}^{2}}+\frac{4 R_{p}^{3}}{15 k_{\text {ext }} D_{\text {eff }}}+\frac{2 R_{p}^{2}}{3 k_{\text {ext }}^{2}}\right]\left[\left(4+6 a^{*} F\right)\left(1-\frac{D_{L}}{L u}\left(1-e^{\left.-\frac{L u}{D_{L}}\right)}\right)+\frac{a^{*} F L u}{D_{L}}\right]\right. \\
& +\frac{8 L F a^{* 4}}{u}\left(\frac{1}{525} \frac{R_{p}^{6}}{D_{e f f}^{3}}+\frac{3}{175} \frac{R_{p}^{5}}{D_{e f f}^{2} k_{e x t}}+\frac{1}{15} \frac{R_{p}^{4}}{D_{e f f} k_{e x t}^{2}}+\frac{1}{9} \frac{R_{p}^{3}}{k_{e x t}^{3}}\right)-\frac{4}{175} \frac{L F^{2} D_{L} a^{* 4} R_{p}^{4}}{u^{3} D_{e f f}^{2}}\left[2+\frac{L u}{D_{L}}\right] \\
& +\frac{t_{i n j}^{4}}{80}+t_{i n j}^{2} \frac{D_{L}^{2}\left(1+a^{*} F\right)^{2}}{u^{4}}\left[\frac{L u}{D_{L}}+e^{-\frac{L u}{D_{L}}}-1\right]+t_{i n j}^{2} \frac{L F}{u}\left[\frac{R_{p} a^{* 2}}{3 k_{e x t}}+\frac{R_{p}^{2} a^{* 2}}{15 D_{e f f}}\right] .(\mathrm{B}-23)
\end{aligned}
$$

In order to calculate the fourth central moment of continuous breakthrough profiles, Eq.

(B-23) reduces as

$$
\begin{aligned}
\mu_{4}^{\prime}= & \frac{12 D_{L}^{4}\left(1+a^{*} F\right)^{4}}{u^{8}}\left[2 e^{-\frac{2 L u}{D_{L}}}+\left(4 \frac{L^{2} u^{2}}{D_{L}^{2}}+22 \frac{L u}{D_{L}}+26\right) e^{-\frac{L u}{D_{L}}}+\frac{L^{2} u^{2}}{D_{L}^{2}}+8 \frac{L u}{D_{L}}-28\right] \\
& +\frac{24 D_{L}^{3}\left(1+a^{*} F\right)^{2} F}{u^{6}}\left(\frac{R_{p} a^{* 2}}{3 k_{e x t}}+\frac{R_{p}^{2} a^{* 2}}{15 D_{\text {eff }}}\right)\left[\frac{L^{2} u^{2}}{D_{L}^{2}}+5 \frac{L u}{D_{L}}-12+\left(7 \frac{L u}{D_{L}}+12\right) e^{-\frac{L u}{D_{L}}}\right] \\
& +\frac{2 L D_{L} F a^{* 3}}{u^{3}}\left[\frac{4 R_{p}^{4}}{105 D_{\text {eff }}^{2}}+\frac{4 R_{p}^{3}}{15 k_{\text {ext }} D_{\text {eff }}}+\frac{2 R_{p}^{2}}{3 k_{\text {ext }}^{2}}\right]\left[\left(4+6 a^{*} F\right)\left(1-\frac{D_{L}}{L u}\left(1-e^{-\frac{L u}{D_{L}}}\right)\right)+\frac{a^{*} F L u}{D_{L}}\right] \\
& +\frac{8 L F a^{* 4}}{u}\left(\frac{1}{525} \frac{R_{p}^{6}}{D_{\text {eff }}^{3}}+\frac{3}{175} \frac{R_{p}^{5}}{D_{\text {eff }}^{2} k_{\text {ext }}}+\frac{1}{15} \frac{R_{p}^{4}}{D_{\text {eff }} k_{\text {ext }}^{2}}+\frac{1}{9} \frac{R_{p}^{3}}{k_{\text {ext }}^{3}}\right) \\
& -\frac{4}{175} \frac{L F^{2} D_{L} a^{* 4} R_{p}^{4}}{u^{3} D_{\text {eff }}^{2}}\left[2\left(1-\frac{D_{L}}{L u}\left(1-e^{-\frac{L u}{D_{L}}}\right)\right)+\frac{L u}{D_{L}}\right] .
\end{aligned}
$$

\section{Appendix C}

\section{Relation between LKM and GRM}

The analytical solutions and moment analysis for the LKM and EDM are given in detail in

the earlier article by Javeed et al. (2013). Here, the relationship between GRM and LKM are derived with respect to moments considering the Danckwerts BCs (c.f. Eq. (12a)). 
The first moments indicating retention times must be identical for LKM and GRM. This is fulfilled provided the following relation holds

$$
a=a^{*} \text {. }
$$

On matching the second central moments of GRM given by Eq. (B-6) and LKM given in Javeed et al. (2013), we obtain the following relation between LKM and GRM parameters

$$
k_{L K M}=\left[\frac{a^{*}}{1-\epsilon}\left(\frac{R_{p}}{3 k_{\text {ext }}}+\frac{R_{p}^{2}}{15 D_{\text {eff }}}\right)\right]^{-1} .
$$

For this value of $K_{L K M}$, the second central moments of GRM and $L K M$ are the same but all higher moments are different.

On equating the third central moments of LKM (see Javeed et al. (2013)) and GRM (c.f. Eq. (B-9)), the following relation is obtianed for $k_{L K M}$ :

$$
k_{L K M}=\frac{\alpha+\sqrt{\alpha^{2}+4 \beta(\alpha \gamma+\delta)}}{2(\alpha \gamma+\delta)},
$$

where

$$
\begin{aligned}
& \alpha=\frac{12 L D_{L} a^{*} \epsilon F^{2}\left(1+a^{*} F\right)}{u^{3}}\left[\frac{D_{L}}{L u}\left(e^{-\frac{L u}{D_{L}}}-1\right)+1\right], \quad \beta=\frac{L a^{*} \epsilon^{2} F^{3}}{u}, \\
& \gamma=\frac{a^{*}}{\epsilon F}\left(\frac{R_{p}}{3 k_{\text {ext }}}+\frac{R_{p}^{2}}{15 D_{\text {eff }}}\right), \quad \delta=\frac{L a^{* 3} F}{u}\left(\frac{4 R_{p}^{4}}{105 D_{\text {eff }}^{2}}+\frac{4 R_{p}^{3}}{15 k_{\text {ext }} D_{\text {eff }}}+\frac{2 R_{p}^{2}}{3 k_{\text {ext }}^{2}}\right) .
\end{aligned}
$$

With this value of $k_{L K M}$ both models have identical third central moment and all other moments are different except $\mu_{1}$.

On matching the fourth central moments of LKM and GRM, we obtain the following relation for $k_{L K M}$ :

$$
k_{L K M}=\frac{1}{3 \xi}\left[\alpha^{\prime}-\nu \zeta-\frac{\Delta_{0}}{\nu \zeta}\right], \quad \text { for } \zeta=\sqrt[3]{\frac{\Delta_{1}+\sqrt{\Delta_{1}^{2}-4 \Delta_{0}^{3}}}{2}} \text { and } \nu=\frac{-1+i \sqrt{3}}{2} .
$$


Moreover,

$$
\begin{aligned}
\Delta_{0}= & \alpha^{\prime 2}+3 \xi \eta, \quad \Delta_{1}=-2 \alpha^{\prime 3}-9 \xi \alpha^{\prime} \eta-27 \xi^{2} \beta^{\prime}, \\
\alpha^{\prime}= & \frac{24 D_{L}^{3} a^{*} \epsilon F^{2}\left(1+a^{*} F\right)^{2}}{u^{6}}\left[\frac{L^{2} u^{2}}{D_{L}^{2}}+5 \frac{L u}{D_{L}}-12+\left(7 \frac{L u}{D_{L}}+12\right) e^{-\frac{L u}{D_{L}}}\right], \\
\beta^{\prime}= & \frac{24 L \epsilon^{3} a^{*} F^{4}}{u}, \quad \eta=\frac{\beta^{\prime} D_{L}}{2 \epsilon F u^{2}}\left[\left(4+6 a^{*} F\right)\left(1-\frac{D_{L}}{L u}\left(1-e^{-\frac{L u}{D_{L}}}\right)\right]+\frac{a^{*} F L u}{D_{L}}\right], \\
\phi= & \frac{8 L F a^{* 4}}{u}\left(\frac{1}{525} \frac{R_{p}^{6}}{D_{e f f}^{3}}+\frac{3}{175} \frac{R_{p}^{5}}{D_{e f f}^{2} k_{e x t}}+\frac{1}{15} \frac{R_{p}^{4}}{D_{e f f} k_{e x t}^{2}}+\frac{1}{9} \frac{R_{p}^{3}}{k_{e x t}^{3}}\right) \\
& -\frac{4}{175} \frac{L F^{2} D_{L} a^{* 4} R_{p}^{4}}{u^{3} D_{e f f}^{2}}\left[2\left(1-\frac{D_{L}}{L u}\left(1-e^{-\frac{L u}{D_{L}}}\right)\right)+\frac{L u}{D_{L}}\right], \\
\xi= & \alpha^{\prime} \gamma+\frac{u}{6 L F^{3} \epsilon^{2} a^{*}} \eta \delta+\phi+t_{i n j} \frac{L F}{u}\left(\frac{R_{p}}{3 k_{e x t}}+\frac{R_{p}^{2}}{15 D_{e f f}}\right) .
\end{aligned}
$$

With $k_{L K M}$ in Eq. (C-6), both LKM and GRM have the same $\mu_{4}^{\prime}$ and all other moments are different except $\mu_{1}$.

Acknowledgement: The first author gratefully acknowledge the Higher Education Commission (HEC) of Pakistan for financial support.

\section{References}

Antos, D., Kaczmarski, K., Wojciecha, P., Seidel-Morgenstern, A., 2003. Concentration dependence of lumped mass transfer coefficients: Linear versus non-linear chromatography and isocratic versus gradient operation. Journal of Chromatography A 1006, 61-76.

Carta, G., 1988. Exact analytical solution of a mathematical model for chromatographic operations. Chemical Engineering Science 43, 2877-2883

Danckwerts, P.V., 1953. Continuous flow systems. Chemical Engineering Science 2, 1-9. 
DeCarlo L.T., 1997. On the meaning and use of kurtosis. Psychological Methods 2, 292-307.

Felinger, A., Cavazzini, A., Dondi, F., 2004. Equivalence of the microscopic and macroscopic models of chromatography: stochastic-dispersive versus lumped kinetic model. Journal of Chromatography A 1043, 149-157.

Guiochon, G., 2002. Preparative liquid chromatography. Journal of Chromatography A, $965,129-161$.

Guiochon, G., Lin, B., 2003. Modeling for preparative chromatography, Academic Press.

Guiochon, G., Felinger, A., Shirazi, D.G., Katti, A.M., 2006. Fundamentals of preparative and nonlinear chromatography, 2nd ed. ELsevier Academic press, New York.

Javeed, S., Qamar, S., Seidel-Morgenstern, A., Warnecke, G., 2011. Efficient and accurate numerical simulation of nonlinear chromatographic processes. Computers \& Chemical Engineering 35, 2294-2305.

Javeed, S., Qamar, S., Seidel-Morgenstern, A., Warnecke, G., 2011. A discontinuous Galerkin method to solve chromatographic models. Journal of Chromatography A 1218, $7137-7146$.

Javeed, S., Qamar, S., Ashraf, W., Seidel-Morgenstern, A., Warnecke, G., 2013. Analysis and numerical investigation of two dynamic models for liquid chromatography. Chemical Engineering Science 90, 17-31.

Qamar, S., Abbasi, J.N., Javeed, S., Shah, M., Khan, F.U., Seidel-Morgenstern, A., 2013. 
Analytical solutions and moment analysis of chromatographic models for rectangular pulse injections, Journal of Chromatography A 1315, 92-106.

Kubin, M., 1965. Beitrag zur Theorie der Chromatographie. Collection of Czechoslovak Chemical Communications 30, 1104-1118.

Kubin, M., 1965. Beitrag zur Theorie der Chromatographie. 11. Einfluss der Diffusion Ausserhalb und der Adsorption Innerhalb des Sorbens- Korns. Collection of Czechoslovak Chemical Communications 30, 2900-2907.

Kucera, E., 1965. Contribution to the theory of chromatography : Linear non-equilibrium elution chromatography. Journal of Chromatography A 19, 237-248.

Lenhoff, A.M., 1987. Significance and estimation of chromatographic parameters. Journal of Chromatography A 384, 285-299.

Miyabe, K., Guiochon, G., 2000. Influence of the modification conditions of alkyl bonded ligands on the characteristics of reversed-phase liquid chromatography. Journal of Chromatography A 903, 1-12.

Miyabe, K., Guiochon, G., 2003. Measurement of the parameters of the mass transfer kinetics in high performance liquid chromatography. Journal of separation science 26, $155-173$.

Miyabe, K., 2007. Surface diffusion in reversed-phase liquid chromatography using silica gel stationary phases of different C1 and C18 ligand densities. Journal of Chromatography A 1167, 161-170. 
Miyabe, K., 2009. Moment analysis of chromatographic behavior in reversed-phase liquid chromatography. Journal of separation science 32, 757-770.

Rice, R.G., Do, D.D., 1995. Applied Mathematics and Modeling for Chemical Engineers, Wiley-Interscience, New York.

Ruthven, D.M., 1984. Principles of adsorption and adsorption processes, WileyInterscience, New York.

Schneider, P., Smith, J.M., 1968. Adsorption rate constants from chromatography. A.I.Ch.E. Journal 14, 762-771.

Seidel-Morgenstern, A., 1991. Analysis of boundary conditions in the axial dispersion model by application of numerical Laplace inversion. Chemical Engineering Science 46, 25672571.

Suzuki, M., Smith, J.M., 1971. Kinetic studies by chromatography. Chemical Engineering Scienece 26, 221-235.

Suzuki, M., 1973. Notes on Determining the Moments of the Impulse Response of the Basic Transformed Equations. Journal of Chemical Engineering of Japan 6, 540-543.

Van der Laan, Th., 1958. Letter to the Editors on Notes on the diffusion type model for the longitudinal mixing in flow. Chemical Engineering Science 7, 187-191.

Van Genuchten, M.Th., 1981. Analytical solutions for chemical transport with simultaneous 
adsorption, zerot-order production and first order decay. Journal of Hydrology 49, 213233.

Wolff, H.-J., Radeke, K.-H, Gelbin, D., 1980. Heat and mass transfer in packed beds-IV use of weighted moments to determine axial dispersion coefficient. Chemical Engineering Science 34, 101-107.

Wolff, H.-J., Radeke, K.-H, Gelbin, D., 1980. Weighted moments and the pore-diffusion model. Chemical Engineering Science 35, 1481-1485. 


\begin{tabular}{|c|c|}
\hline Nom & \\
\hline$a$ & Henry constant [-] \\
\hline$B i$ & Biot number [-] \\
\hline$c$ & liquid phase concentration, $[g / l]$ \\
\hline$c_{p}$ & concentration in particle pores, $[g / l]$ \\
\hline$d$ & column diameter, $[m]$ \\
\hline$D_{L}$ & apparent dispersion coefficient $\left[\mathrm{cm}^{2} / \mathrm{min}\right]$ \\
\hline$D_{p}$ & pore diffusivity $\left[\mathrm{cm}^{2} / \mathrm{min}\right]$ \\
\hline$D_{s}$ & surface diffusivity $\left[\mathrm{cm}^{2} / \mathrm{min}\right]$ \\
\hline$D_{e f f}$ & effective diffusion coefficient $\left[\mathrm{cm}^{2} / \mathrm{min}\right]$ \\
\hline$k_{e x t}$ & external transfer coefficient, $[1 / \min ]$ \\
\hline$k_{L K M}$ & mass transfer coefficient used in LKM, $[1 / \min ]$ \\
\hline$L$ & column length, $[\mathrm{cm}]$ \\
\hline$N$ & no of theoretical plates, $[-]$ \\
\hline$P e$ & Peclet number, [-] \\
\hline$q^{*}$ & solid phase concentration, $[\mathrm{mol} / \mathrm{l}]$ \\
\hline$R_{p}$ & radius of spherical particles, $[\mathrm{cm}]$ \\
\hline$r$ & radial coordinate of spherical particle of radius $R_{p},[\mathrm{~cm}]$ \\
\hline$t$ & time, $[s]$ \\
\hline$u$ & interstitial velocity, $[\mathrm{m} / \mathrm{s}]$ \\
\hline$z$ & spatial coordinate, $[\mathrm{cm}]$ \\
\hline
\end{tabular}




$\begin{array}{ll}\text { Nomenclature (continued) } & \\ \epsilon & \text { external porosity } \\ \epsilon_{p} & \text { internal porosity } \\ \mu_{n} & n \text {-th initial normalized moment } \\ \mu_{n}^{\prime} & n \text {-th central moment } \\ \text { Subscripts } & \\ \text { inj } & \text { injection } \\ \text { Abbreviations } & \\ \text { BCs } & \text { lumped kinetic model } \\ \text { EDM } & \text { finite volume scheme } \\ \text { FVS } & \text { goundary conditions } \\ \text { LRM } & \text { equilibrium dispersive model } \\ & \\ & \end{array}$


Table 1: Analytically determined GRM moments, $\mu_{0}=c_{\mathrm{inj}} t_{\mathrm{inj}}, \mu_{1}=\frac{t_{\mathrm{inj}}}{2}+\frac{L}{u}\left(1+a^{*} F\right)$ and $\mu_{0}=c_{\mathrm{inj}}, \mu_{1}=\frac{L}{u}\left(1+a^{*} F\right)$ for rectangular and breakthrough curves, respectively. The fourth central moment $\mu_{4}^{\prime}$ corresponds to rectangular and continuous breakthrough curves using

Danckwerts and Dirichlet BCs are given in Eqs. (B-11), (B-12), (B-23) and (B-24), respectively.

\begin{tabular}{|c|c|c|}
\hline GRM with BCs & $\mu_{2}^{\prime}$ & $\mu_{3}^{\prime}$ \\
\hline $\begin{array}{l}\text { Danckwerts BCs } \\
\text { (Rectangular profiles) }\end{array}$ & $\begin{array}{c}\frac{t_{\mathrm{inj}}^{2}}{12}+\frac{2 L D_{L}\left(1+a^{*} F\right)^{2}}{u^{3}}\left(1+\frac{D_{L}}{L u}\left(e^{-\frac{L u}{D_{L}}}-1\right)\right) \\
+\frac{L F}{u}\left(\frac{2 R_{p} a^{* 2}}{3 k_{\text {ext }}}+\frac{2 R_{p}^{2} a^{* 2}}{15 D_{\text {eff }}}\right)\end{array}$ & $\begin{array}{c}\frac{12 L D_{L}^{2}\left(1+a^{*} F\right)^{3}}{u^{5}}\left[\left(1+\frac{2 D_{L}}{L u}\right) e^{-\frac{L u}{D_{L}}}+\left(1-\frac{2 D_{L}}{L u}\right)\right] \\
+\frac{6 L D_{L} F\left(1+a^{*} F\right)}{u^{3}}\left(\frac{2 R_{p} a^{* 2}}{3 k_{\text {ext }}}+\frac{2 R_{p}^{2} a^{* 2}}{15 D_{\text {eff }}}\right)\left[\frac{D_{L}}{L u}\left(e^{-\frac{L u}{D_{L}}}-1\right)+1\right] \\
+\frac{L a^{* 3} F}{u}\left(\frac{4 R_{p}^{4}}{105 D_{\text {eff }}^{2}}+\frac{4 R_{p}^{3}}{15 k_{\text {ext }} D_{\text {eff }}}+\frac{2 R_{p}^{2}}{3 k_{\text {ext }}^{2}}\right)\end{array}$ \\
\hline $\begin{array}{l}\text { Danckwerts BCs } \\
\text { (Breakthrough curves) }\end{array}$ & $\begin{array}{l}\frac{2 L D_{L}\left(1+a^{*} F\right)^{2}}{u^{3}}\left(1+\frac{D_{L}}{L u}\left(e^{-\frac{L u}{D_{L}}}-1\right)\right) \\
\quad+\frac{L F}{u}\left(\frac{2 R_{p} a^{* 2}}{3 k_{e x t}}+\frac{2 R_{p}^{2} a^{* 2}}{15 D_{\text {eff }}}\right)\end{array}$ & same as GRM (Rectangular profiles) \\
\hline $\begin{array}{l}\text { Dirichlet BCs } \\
\text { (Rectangular profiles) }\end{array}$ & $\begin{aligned} \frac{t_{\mathrm{inj}}^{2}}{12}+ & \frac{L F}{u}\left(\frac{2 R_{p} a^{* 2}}{3 k_{\text {ext }}}+\frac{2 R_{p}^{2} a^{* 2}}{15 D_{\text {eff }}}\right) \\
& +\frac{2 L D_{L}}{u^{3}}\left(1+a^{*} F\right)^{2}\end{aligned}$ & $\begin{array}{c}\frac{6 L D_{L}\left(1+a^{*} F\right) F}{u^{3}}\left(\frac{2 R_{p} a^{* 2}}{3 k_{e x t}}+\frac{2 R_{p}^{2} a^{* 2}}{15 D_{e f f}}\right) \\
+\frac{L F a^{* 3}}{u}\left(\frac{4 R_{p}^{4}}{105 D_{\text {eff }}^{2}}+\frac{4 R_{p}^{3}}{15 k_{e x t} D_{\text {eff }}}+\frac{2 R_{p}^{2}}{3 k_{\text {ext }}^{2}}\right) \\
+\frac{12 L D_{L}^{2}}{u^{5}}\left(1+a^{*} F\right)^{3}\end{array}$ \\
\hline $\begin{array}{l}\text { GRM Dirichlet BCs } \\
\text { (Breakthrough curves) }\end{array}$ & $\frac{L F}{u}\left(\frac{2 R_{p} a^{* 2}}{3 k_{e x t}}+\frac{2 R_{p}^{2} a^{* 2}}{15 D_{\text {eff }}}\right)+\frac{2 L D_{L}}{u^{3}}\left(1+a^{*} F\right)^{2}$ & same as GRM (Rectangular profiles) \\
\hline
\end{tabular}


Table 2: Parameters of the problems.

\begin{tabular}{|c|c|}
\hline Parameters & values \\
\hline Column length & $L=1.7 \mathrm{~cm}$ \\
Pore radius & $R_{p}=0.004 \mathrm{~cm}$ \\
External porosity & $\epsilon=0.4$ \\
Internal porosity & $\epsilon_{p}=0.333$ \\
External mass transfer coefficient & $k_{\text {ext }}=0.01 \mathrm{~cm} / \mathrm{min}$ \\
Initial concentration & $c(0, z)=0 \mathrm{~g} / \mathrm{l}$ \\
Initial concentration & $c_{p}(0, z)=0 \mathrm{~g} / \mathrm{l}$ \\
Concentration at inlet & $c_{\mathrm{inj}}=1.0 \mathrm{~g} / \mathrm{l}$ \\
Injection time & $t_{\mathrm{inj}}=20 \mathrm{~min}$ \\
Adsorption equilibrium constant & $a=2.5$ \\
\hline
\end{tabular}

Table 3: A comparison of GRM and LKM moments using $a^{*}=a=2.0, u=0.3 \mathrm{~m} / \mathrm{s}, D_{L}=0.002 \mathrm{~cm}^{2} / \mathrm{min}$,

\begin{tabular}{|c|c|c|c|c|}
\hline $\begin{array}{c}k_{L K M} \\
{\left[m i n^{-1}\right]}\end{array}$ & $\begin{array}{c}\mu_{n, G R M}^{\prime}, \mathrm{n}=2,3,4 \\
{\left[\mathrm{~min}^{n}\right]}\end{array}$ & $\begin{array}{l}\mu_{2, L K M}^{\prime} \\
{\left[\min ^{2}\right]}\end{array}$ & $\begin{array}{l}\mu_{3, L K M}^{\prime} \\
{\left[\mathrm{min}^{3}\right]}\end{array}$ & $\begin{array}{l}\mu_{4, L K M}^{\prime} \\
{\left[\min ^{4}\right]}\end{array}$ \\
\hline$k_{L K M}=0.250($ Eq. $(\mathrm{C}-2))$ & $\mu_{2, G R M}^{\prime}=118.94$ & 118.94 & 633.06 & $4.74 \times 10^{4}$ \\
\hline$k_{L K M}=0.217($ Eq. $(\mathrm{C}-3))$ & $\mu_{3, G R M}^{\prime}=831.95$ & 131.36 & 831.95 & $5.98 \times 10^{4}$ \\
\hline$k_{L K M}=0.235($ Eq. $(\mathrm{C}-6))$ & $\mu_{4, G R M}^{\prime}=5.24 \times 10^{4}$ & 124.16 & 713.23 & $5.24 \times 10^{4}$ \\
\hline
\end{tabular}



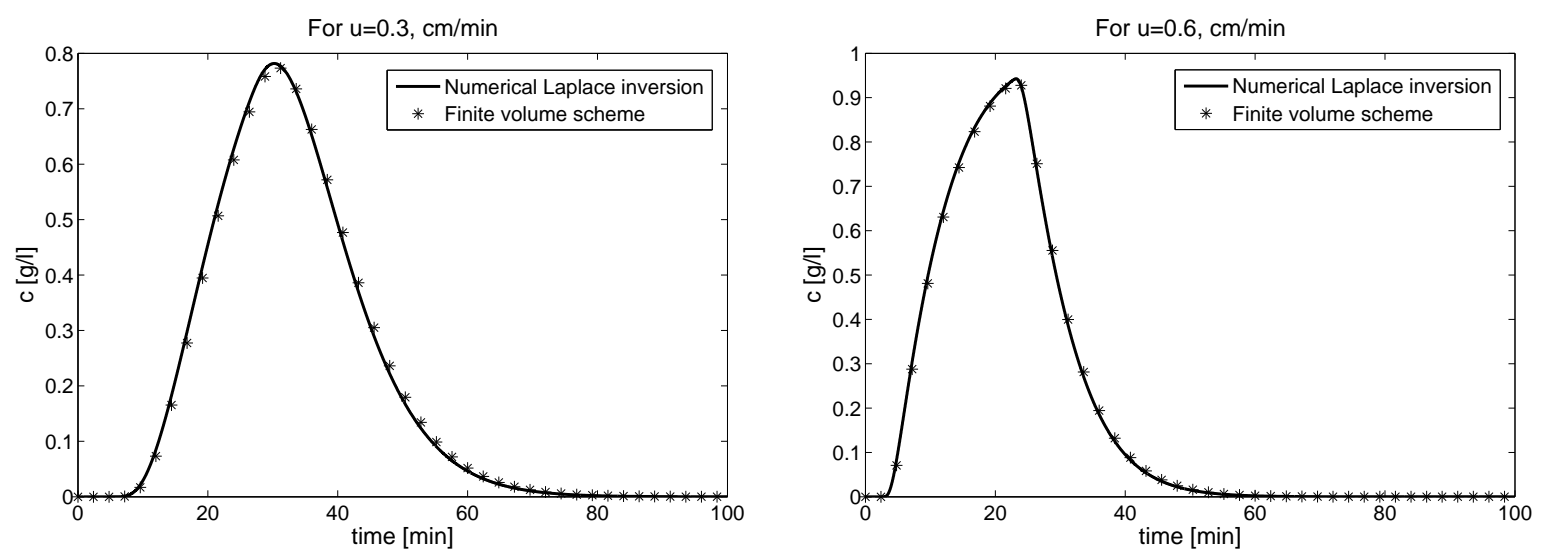

Figure 1: Comparison of solutions obtained by numerical Laplace inversion and FVS for rectangular inlet Danckwerts boundary conditions. Here, $D_{L}=0.002 \mathrm{~cm}^{2} / \mathrm{min}, D_{\text {eff }}=10^{-6} \mathrm{~cm}^{2} / \mathrm{min}$ and other parameters are given in Table 2.
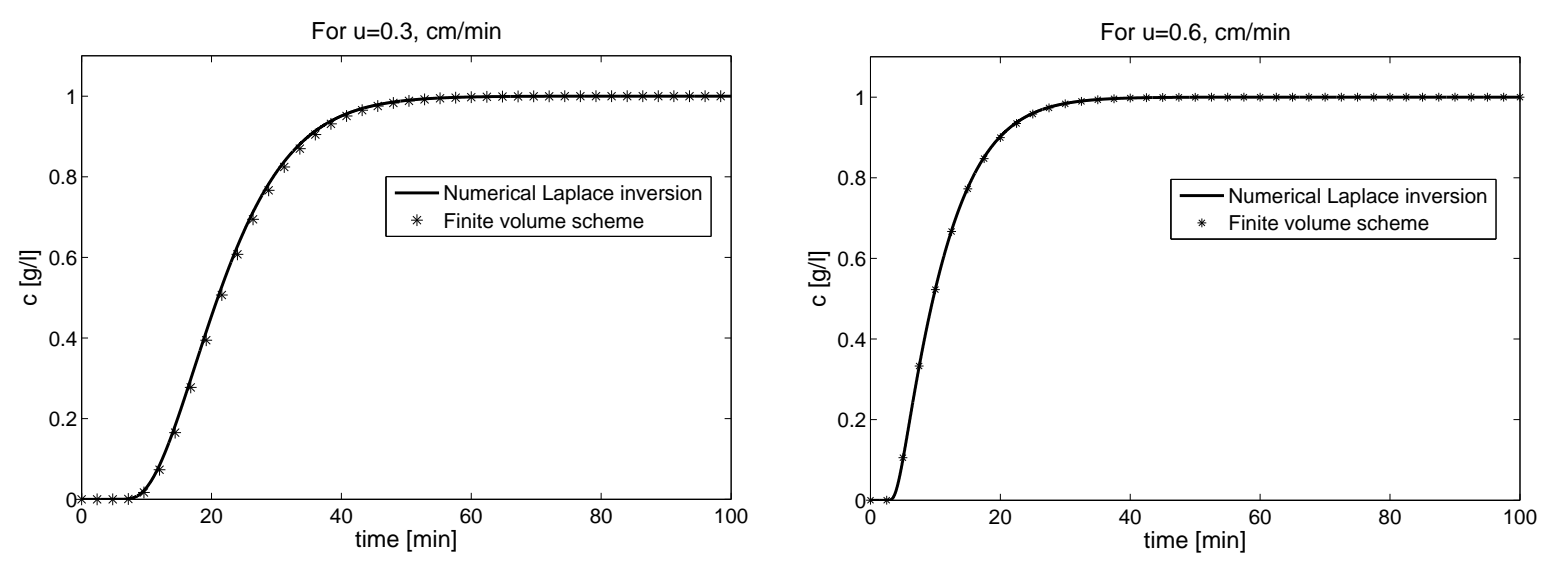

Figure 2: Comparison of solutions obtained by numerical Laplace inversion and FVS for continuous breakthrough inlet Danckwerts boundary conditions. Here, $D_{L}=0.002 \mathrm{~cm}^{2} / \min , D_{\text {eff }}=10^{-6} \mathrm{~cm}^{2} / \min$ and other parameters are given in Table 2 . 

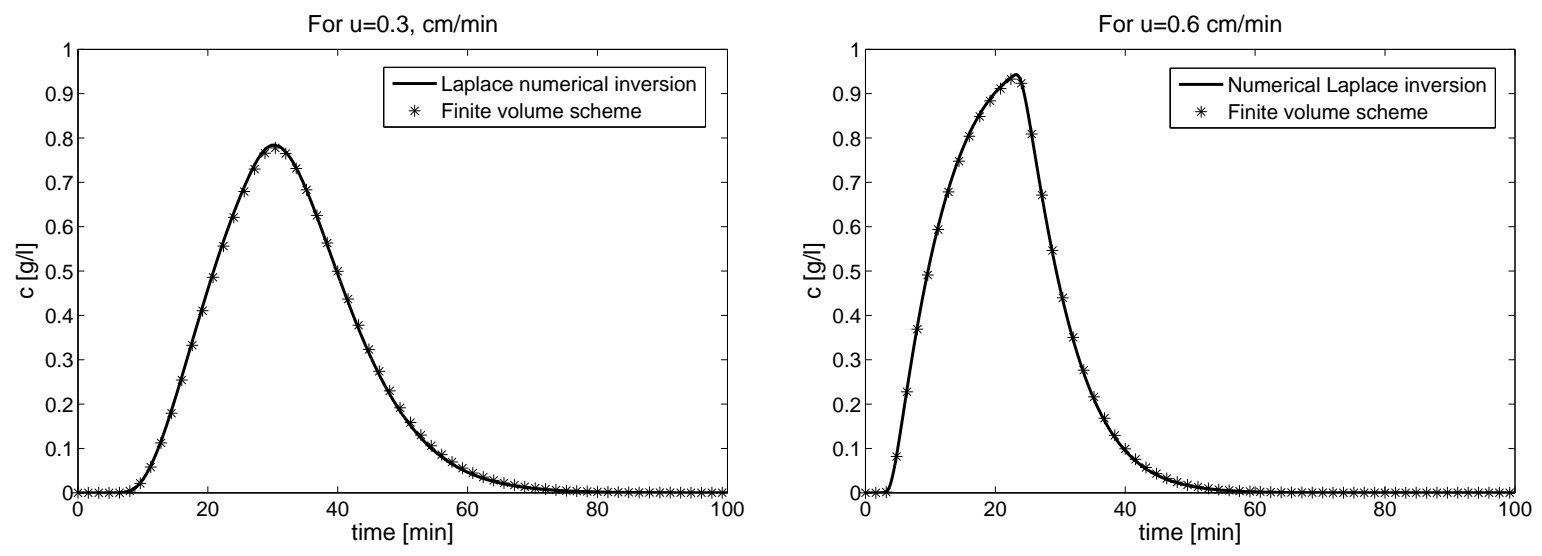

Figure 3: Comparison of solutions obtained by numerical Laplace inversion and FVS for rectangular inlet Dirichlet boundary conditions. Here, $D_{L}=0.002 \mathrm{~cm}^{2} / \mathrm{min}, D_{\text {eff }}=10^{-6} \mathrm{~cm}^{2} / \mathrm{min}$ and other parameters are given in Table 2 .
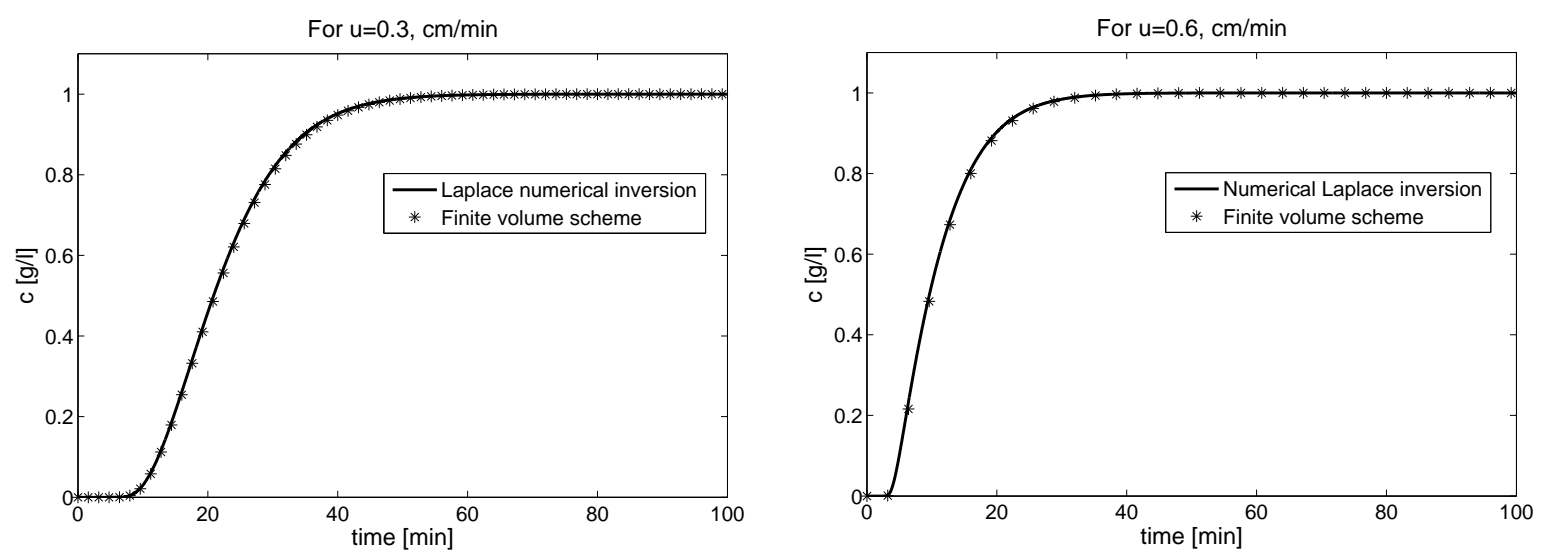

Figure 4: Comparison of solutions obtained by numerical Laplace inversion and FVS for continuous breakthrough inlet Dirichlet boundary conditions. Here, $D_{L}=0.002 \mathrm{~cm}^{2} / \mathrm{min}, D_{\text {eff }}=10^{-6} \mathrm{~cm}^{2} / \mathrm{min}$ and other parameters are given in Table 2 . 


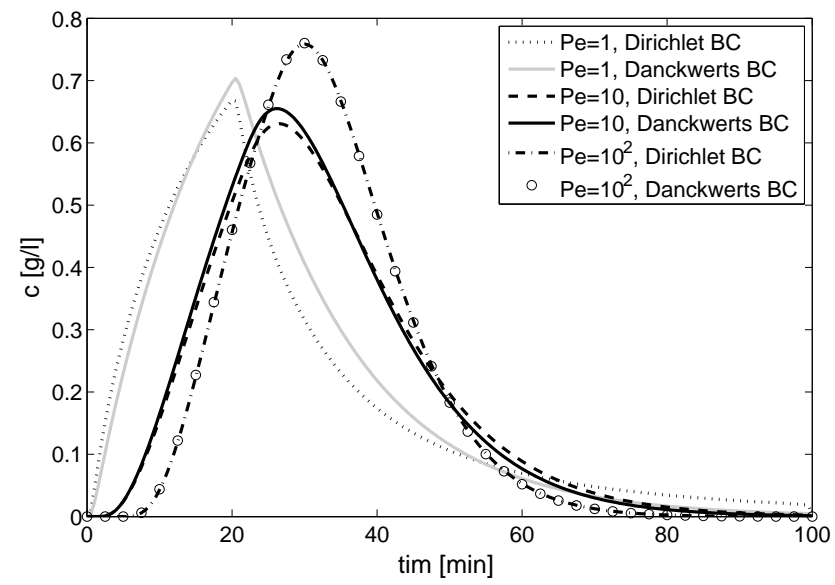

Figure 5: Effects of Dirichlet and Danckwerts boundary conditions for two Peclet numbers using numerical Laplace inversion solution. Here, $u=0.3 \mathrm{~cm} / \mathrm{min}, D_{\text {eff }}=10^{-6} \mathrm{~cm}^{2} / \mathrm{min}$ and other parameters are given in Table 2 .
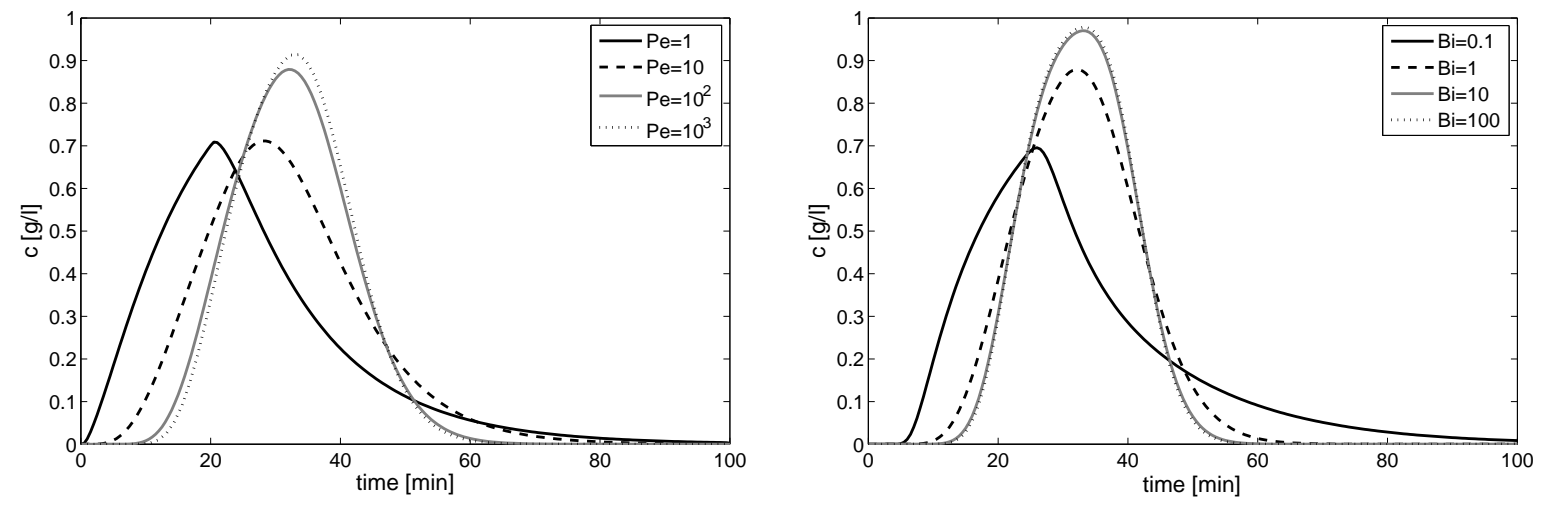

Figure 6: Effects of Peclet number $P e$ for Biot number $B i=1$ and $B i$ for $P e=100$. Here, $u=0.3 \mathrm{~cm} / \mathrm{min}$ and other parameters are given in Table 2. 

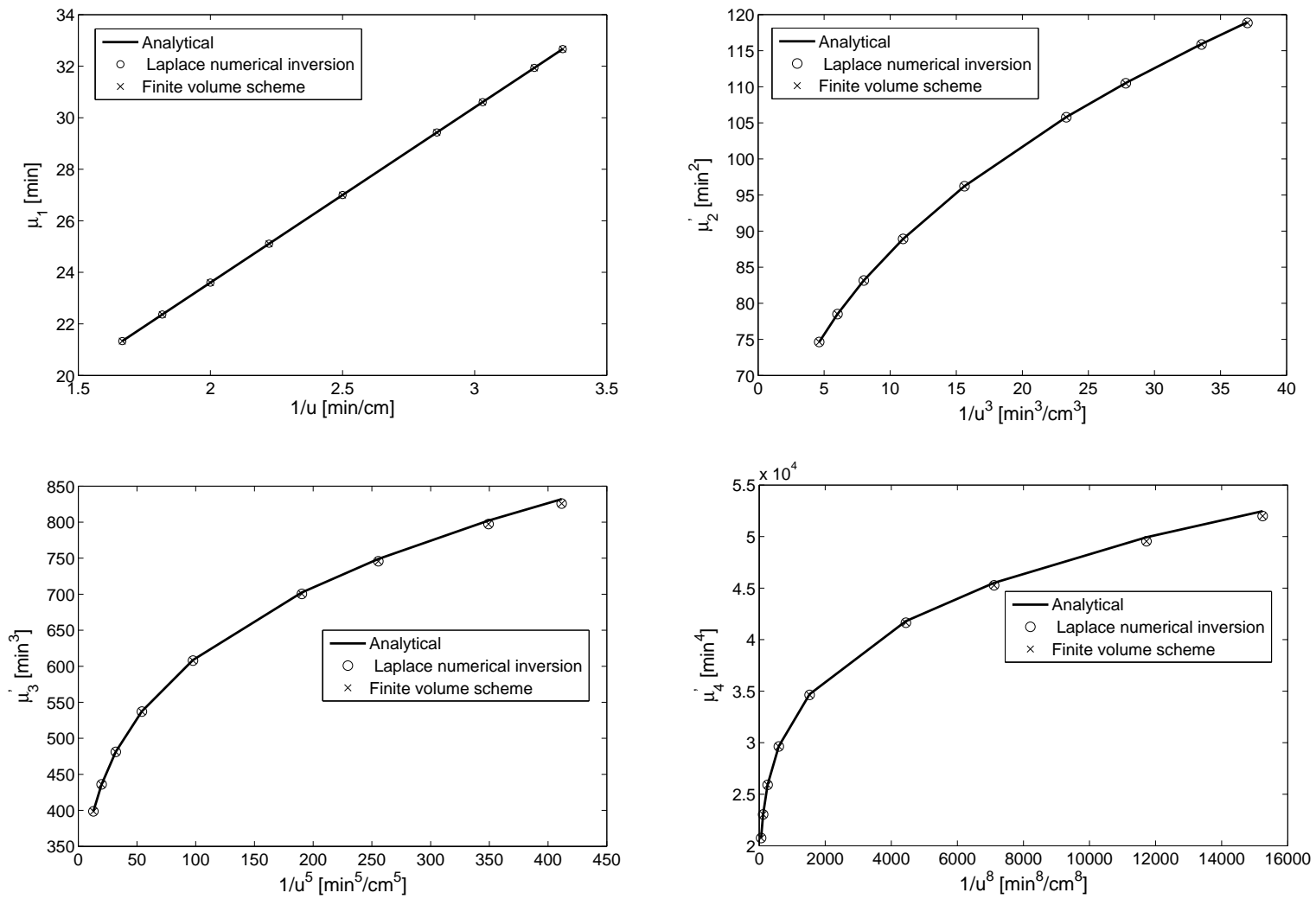

Figure 7: Comparison of analytical and numerical moments for rectangular inlet Danckwerts BCs. Here, $D_{L}=0.002 \mathrm{~cm}^{2} / \min , D_{\text {eff }}=10^{-6} \mathrm{~cm}^{2} / \mathrm{min}$ and other parameters are given in Table 2. 

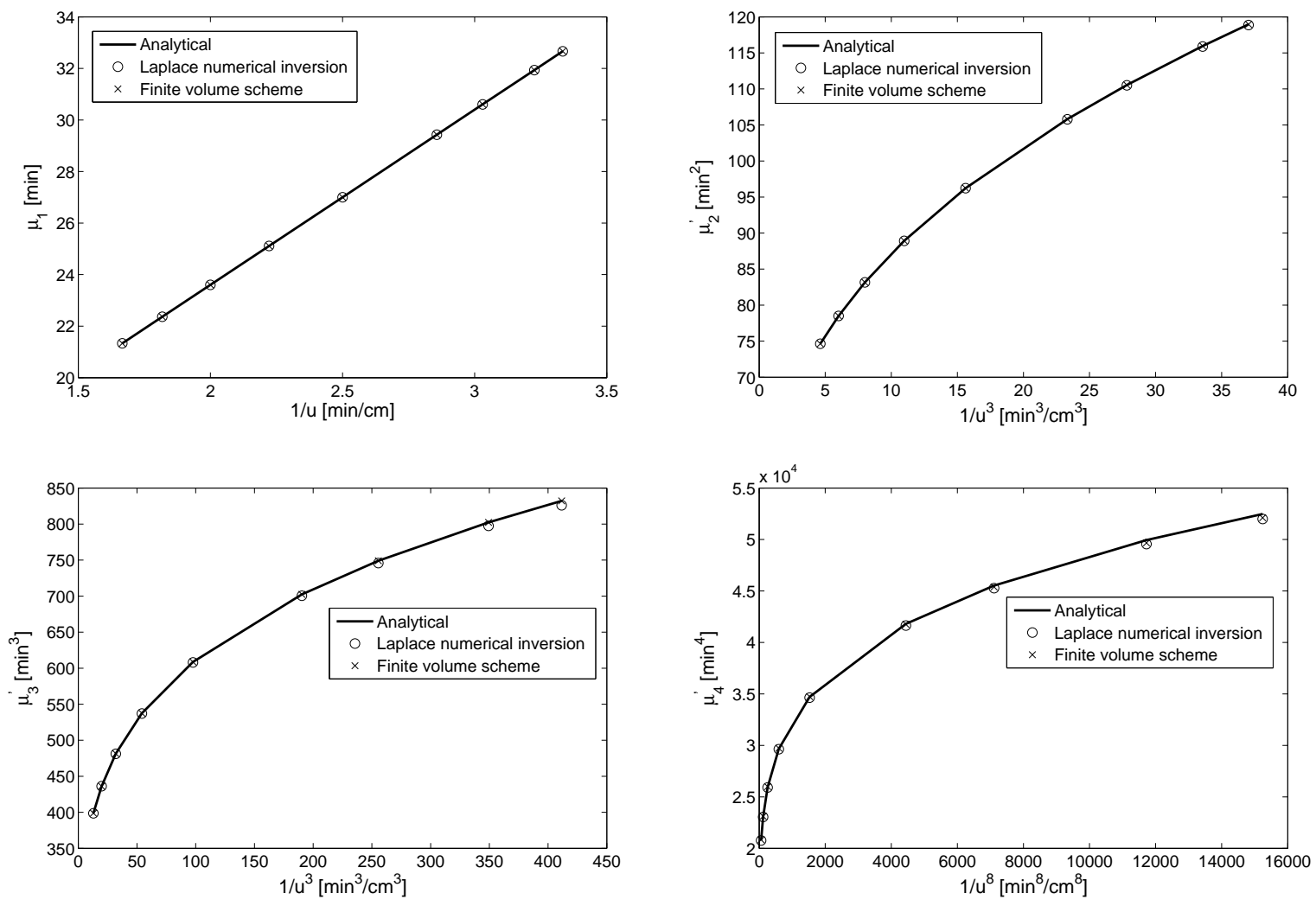

Figure 8: Comparison of analytical and numerical moments for rectangular inlet Dirichlet BCs. Here, $D_{L}=0.002 \mathrm{~cm}^{2} / \min , D_{\text {eff }}=10^{-6} \mathrm{~cm}^{2} / \min$ and other parameters are given in Table 2.

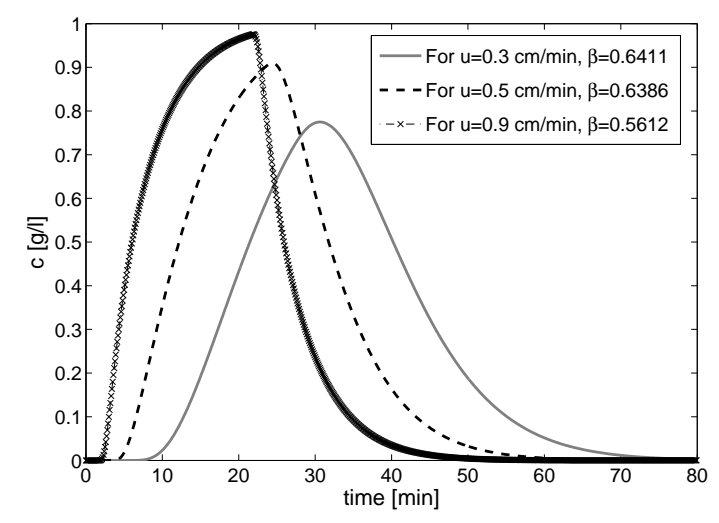

Figure 9: Skewness associated with third central moments $\mu_{3}^{\prime}$ considering different velocities $u$ with $D_{L}=$ $0.002 \mathrm{~cm}^{2} / \min$ and $D_{\text {eff }}=10^{-6} \mathrm{~cm}^{2} / \min$. All other parameters are given in Table 2 . 


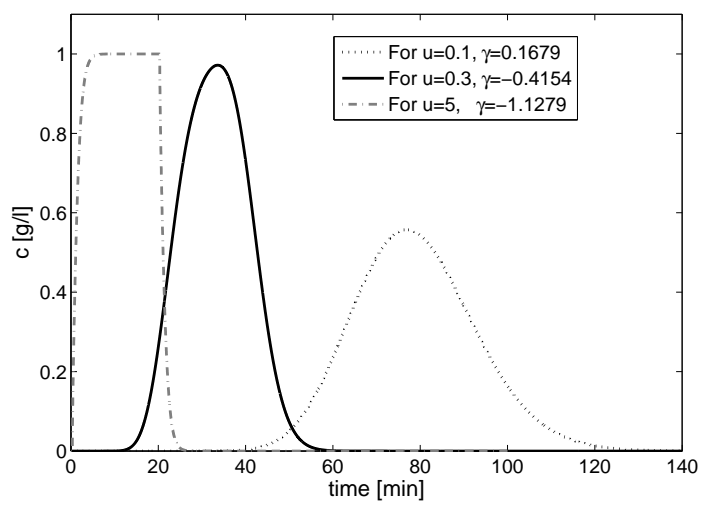

Figure 10: The fourth central moments $\mu_{4}^{\prime}$ related to kurtosis for different velocities $u$ with $D_{L}=$ $0.002 \mathrm{~cm}^{2} /$ min and $D_{\text {eff }}=6.3835 \times 10^{-5} \mathrm{~cm}^{2} / \mathrm{min}$. All other parameters are given in Table 2 . 

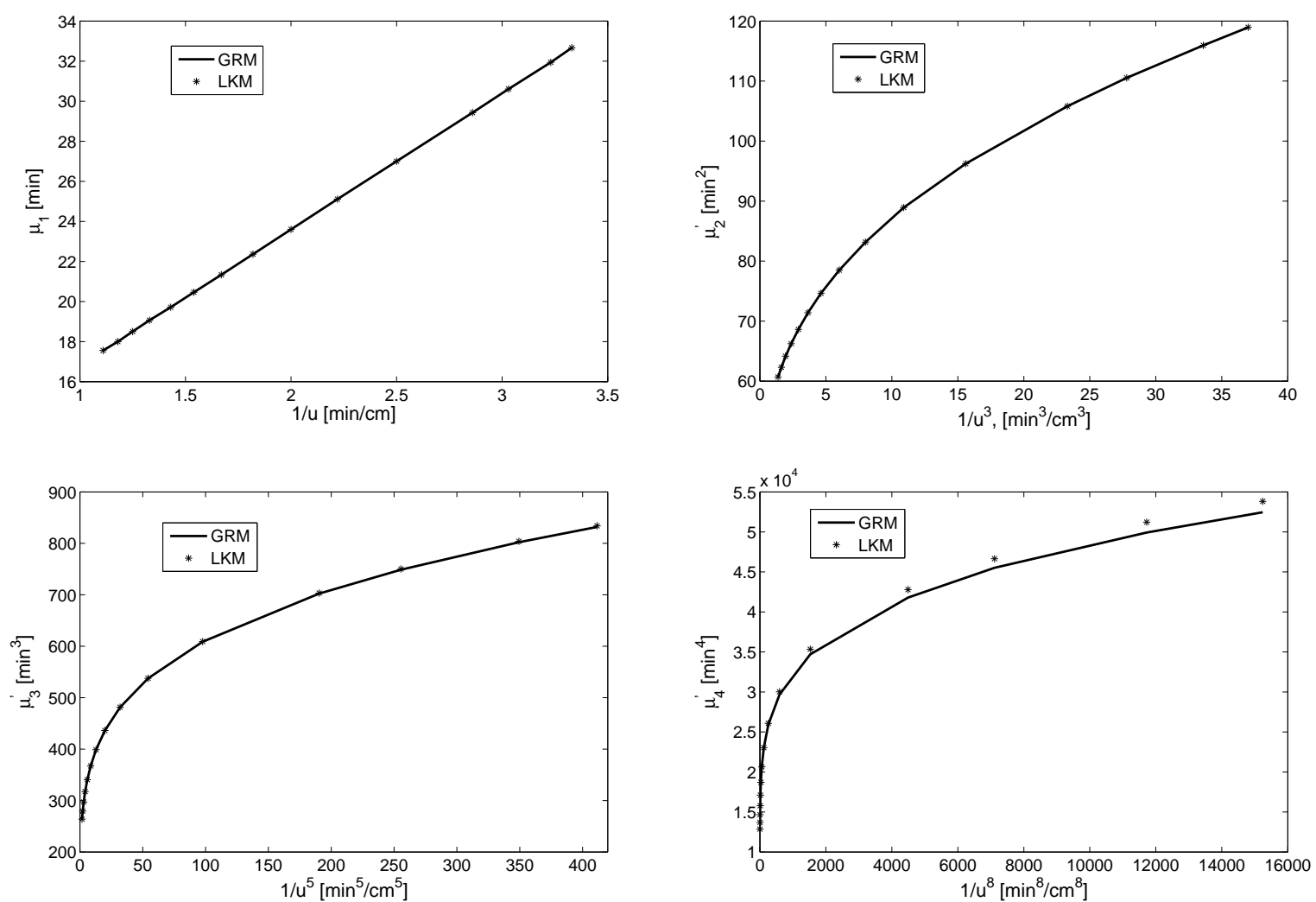

Figure 11: Moments agreement of LKM and GRM when parameters were matched through relations in Eqs. (C-2), (C-3) and (C-6). Here, $D_{L}=0.002 \mathrm{~cm}^{2} / \mathrm{min}, D_{\text {eff }}=10^{-6} \mathrm{~cm}^{2} / \mathrm{min}, u=0.3 \mathrm{~cm} / \mathrm{min}$ and other parameters are given in Table 2 . 


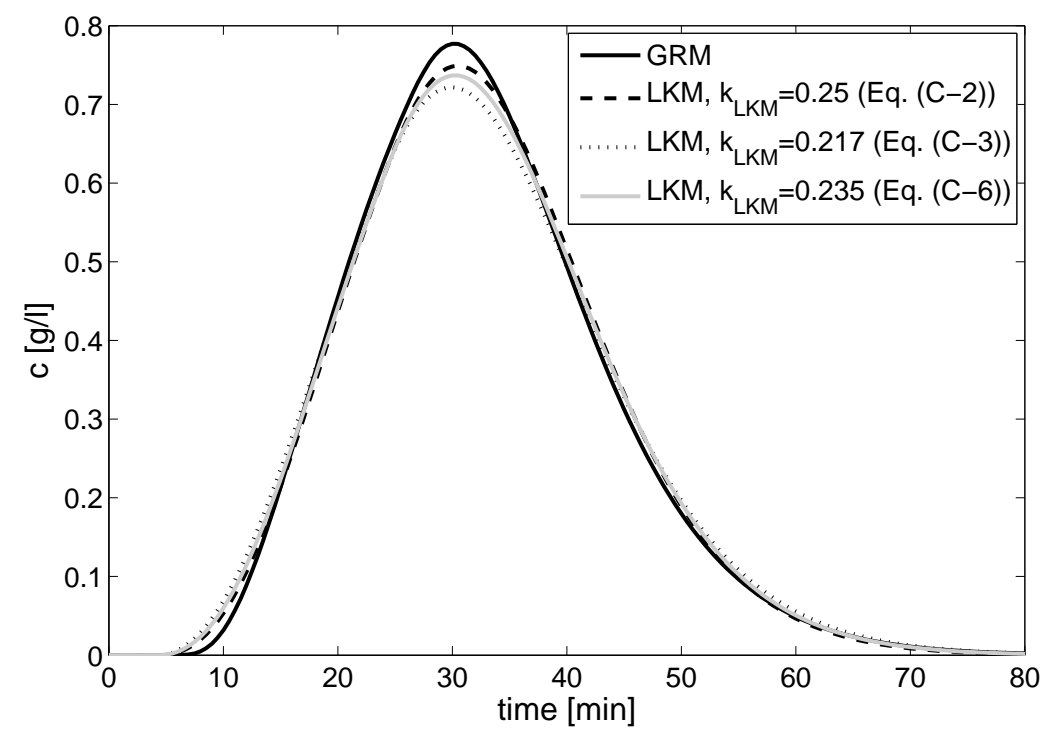

Figure 12: Comparison of solution profiles from GRM and LKM using different values of $k_{L K M}$ obtained from the three expressions given by Eqs. (C-2), (C-3) and (C-6). Here, $u=0.3 \mathrm{~cm} / \mathrm{min}$, $D_{L}=0.002 \mathrm{~cm}^{2} / \min , D_{\text {eff }}=10^{-6} \mathrm{~cm}^{2} / \mathrm{min}$ and other parameters are given in Table 2. 